\title{
FUlL-COLOR III-NitRIDE NANOWIRE Light-EMitTing Diodes
}

\author{
Ravi Teja VELPULA, Barsha JAIN, Ha Quoc Thang BUI, \\ Hieu Pham Trung NGUYEN*
}

Department of Electrical and Computer Engineering, New Jersey Institute of Technology, 323 Dr Martin Luther King Jr Boulevard, Newark, New Jersey, 07102, U.S.A.

*Corresponding Author: Hieu Pham Trung NGUYEN (Email: hieu.p.nguyen@njit.edu)

(Received: 03-Dec-2018; accepted: 26-Dec-2019; published: 31-Dec-2019)

DOI: http://dx.doi.org/10.25073/jaec.201934.271

\begin{abstract}
III-nitride nanowire based lightemitting diodes (LEDs) have been intensively studied as promising candidates for future lighting technologies. Compared to conventional GaN-based planar LEDs, III-nitride nanowire LEDs exhibit numerous advantages including greatly reduced dislocation densities, polarization fields, and quantum-confined Stark effect due to the effective lateral stress relaxation, promising high efficiency full-color LEDs. Beside these advantages, however, several issues have been identified as the limiting factors for

will describe the current methods for the fabrication of nanowire structures including top-down and bottom-up approaches, followed by characteristics of III-nitride nanowire LEDs. We will then discuss the carrier dynamics and loss mechanism in nanowire LEDs. The typical designs for the enhanced performance of III-nitride nanowire LEDs will be presented next. The color tunable nanowire LEDs with emission wavelengths in the visible spectrum, and phosphorfree nanowire white LEDs will be finally discussed.
\end{abstract} further enhancing the nanowire LED quantum efficiency and light output power. Some of the most probable causes have been identified as due to the lack of carrier confinement in the active region, non-uniform carrier distribution, electron overflow, and the nonradiative recombination along the nanowire lateral surfaces. Moreover, the presence of large surface states and defects contribute significantly to the carrier loss in nanowire LEDs. Consequently, reported nanowire LEDs show relatively low output power. Recently, III-nitride core-shell nanowire LED structures have been reported as the most efficient nanowire white LEDs with a record high output power which is more than 500 times stronger than that of nanowire white LEDs without using core-shell structure. In this context, we will review the current status, challenges and approaches for the high performance IIInitride nanowire LEDs. More specifically, we

\section{Keywords}

Light-emitting diodes; Nanowire; nanorod; III-nitride; Molecular beam epitaxy

\section{Fabrication of III-nitride nanowire LEDs}

In the last decade, significant research has been made on the epitaxial growth, fabrication, and characterization of nanowire LEDs. Axial nanowire LEDs can be fabricated using various techniques. Generally, there are two common 
approaches including top-down and bottom-up methods.

\subsection{Top-down nanowires}

Top-down approach utilizes an appropriate etching process via a desired mask to create the nanowire LED from the as-grown planar LED heterostructures. Mask pattern plays an important role to determine the resultant structure formed on wafer surface. Electron beam lithography has been widely used in nanofabrication [1]. This lithographical technique provides a precise direct-writing ability to fabricate uniform nano-patterns, however, time-consuming step and high equipment cost make mass production of this method impractical. Self-assembling of metal nano-islands and other nanospheres are employed to produce large-area mask particles with minimal processing time [2-4]. An alternative candidate, namely nanoimprint lithography, is capable of developing highly ordered nanopatterns over large area in controllable and effective manner while the feature dimensions of the mask patterns strictly rely on the lithographical stamp used. Various etch techniques can be employed after nano-patterning the planar structure, such as inductively coupled plasma (ICP), reactive ion beam (RIE), focus ion beam (FIB) and wet-chemical etching. To minimize the side effects of etching damaged sidewalls of nanostructure, annealing and wet chemical treatments have been proposed to recover plasma-induced defects. Recently, Li et al. demonstrated the fabrication of GaN nanowires by using plasma etching followed by an anisotropic wet etch [4]. Consequently, the nanowires exhibit straight, smooth and well-defined facets. Figure 1.1(a) shows a scanning electron microscopy (SEM) image of a planar LED structure covered with a hexagonal close-packed monolayer of silica spheres. After plasma etching, truncated coneshaped nanowires are formed, as shown in Figure 1.1(b). After the anisotropic wet etch using $\mathrm{KOH}$ based etchant, nanowires with straight and smooth sidewalls are produced. Selectively etching process in which the $n$-type GaN etching is faster than the $p$-type GaN, leading to "flashlight" shaped nanowires, as shown in Figures 1.1(c) and 1.1(d). In their report, they

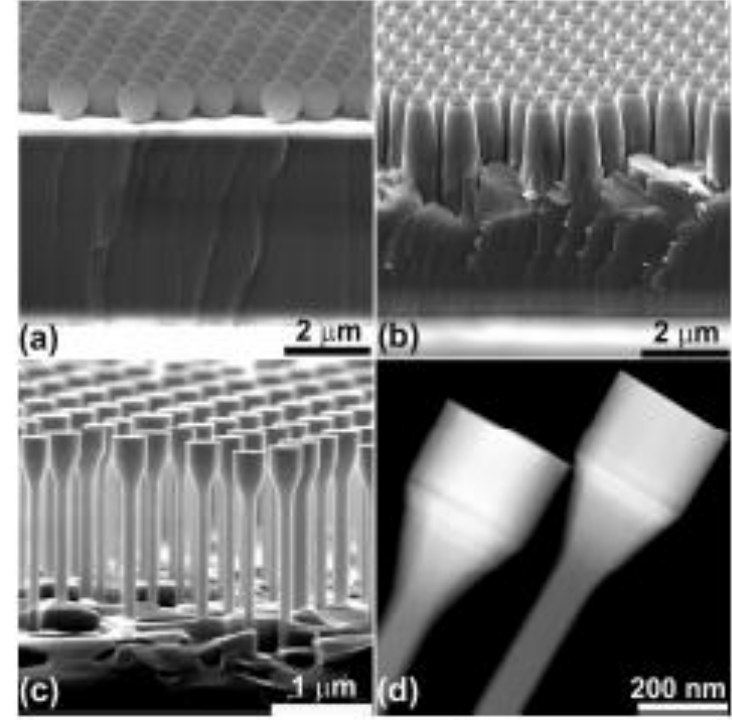

Fig. 1.1: (a) SEM image of an In GaN/GaN planar LED structure covered with a monolayer of silica spheres to serve as a mask for subsequent etching process. (b) Nanowires are formed after plasma etching. (c) After selectively etching process, "flashlight" shaped nanowires with straight and smooth sidewalls were achieved. (d) STEM image of nanowire LEDs [4].

found that $94 \%$ of the nanowires are dislocationfree and a reduced quantum confined Stark effect (QCSE) is observed due to the reduced piezoelectric fields. Bae et al. also demonstrated the size-controlled fabrication of In$\mathrm{GaN} / \mathrm{GaN}$ nanowire arrays by combining a topdown method with a wet treatment process [5]. They found that the photoluminescence (PL) intensity of the as-etched nanowires was the lowest due to plasma-induced damage, while the wettreated nanowires displayed a significantly improved PL intensity. Chiu et al. reported that the wet-treated nanowires displayed enhanced PL intensity, due to the reduced surface damage [2]. For instance, the PL intensity of nanowires made by using ICP and photo-enhanced chemical (PEC) wet oxidation process gained an enhancement of about 6 times compared to that of the as-etch nanowire samples. 


\subsection{Bottom-up nanowires}

In addition of the top-down approach, the bottom-up approach has been investigated. This method overcomes problems associated with top-down method such as the presence of dislocations in the bulk of nanowires and etchinduced defects along the exposed sidewalls. Moreover, the epitaxial growth of current planar LED structures fundamentally restricts the achievement of emission at long wavelength in top-down method due to the high substrate growth temperature. In this regard, nanowire LEDs fabricated by the bottom-up approach have been intensively developed with numerous LED structures including double heterostructures (DH), quantum wells/disks, or quantum dots in the device active region. The nanowire LEDs can be fabricated with emission wavelengths varying from ultraviolet (UV) to the entire visible spectral range. In addition, multicolor nanowire LEDs can be monolithically integrated on a single chip for tunable emission which will be further discussed in the next section.

In the bottom-up method, III-nitride nanowires can be grown by vapor-liquid-solid (VLS), spontaneous formation, and selective area growth (SAG) processes. VLS growth process utilizes metal particles, such as $\mathrm{Au}, \mathrm{Ni}$, and $\mathrm{Fe}$ as catalyst to assist the nucleation and growth of nanowires [6]. The metal particle sizes and densities are carefully controlled to achieve the proper position and the diameter of the nanowires. In addition, growth parameters such as temperature and pressure are very important for the proper growth of nanowires. The VLS growth of III-nitride nanowire structures was performed by using a quartz tube furnace [7], low pressure metal-organic vapor phase epitaxy (MOVPE) [8], thermal chemical vapor deposition (CVD) [9] and low-temperature halide chemical vapor deposition [10]. Generally, in metal catalyst assisted nanowire growth, metal catalyst remains on the tip of nanowire and it may diffuse into semiconductor nanowires during the epitaxial growth, which may introduce impurity in the nanowires. The optical and electrical properties of the related nanostructured LEDs are strongly deteriorated resulted from such unintentionally introduced impurities [11, 12].

III-nitride nanowires can also be grown spontaneously without using any foreign metal catalysts $[13,14]$. Molecular beam epitaxy (MBE) is commonly used for the spontaneous growth of III-nitride nanowires. The growth of GaN nanowires has been demonstrated in this approach on $\mathrm{Si}$ (111) [2] and $\mathrm{Si}$ (001) [17], on AlN [18] and $\mathrm{SiO}_{2}$ [19] buffer layers on $\mathrm{Si}$, and low-temperature GaN [18] buffer layers on $c$ plane sapphire. The V/III ratio plays important role in the MBE growth of self-assembled GaN nanowires. Depending on the growth condition, two different growth regimes (thin film and nanowire) can be clearly identified indicating that adatoms diffusion length and the actual $\mathrm{Ga} / \mathrm{N}$ flux ratios are key factors to achieve nanowires growth.

Practical device applications require higher level of controlling nanowire position and dimension. In this regard, epitaxial growth of nanowires on patterned substrates have been investigated [15-32]. In this growth process, a mask layer is first deposited on the substrate surface. The nano-patterns are then created on the mask for the epitaxial growth of subsequent nanowires. Zarko et al. demonstrated highly uniform GaN nanowires on GaN substrate with $\mathrm{TiN}_{x}$ mask by MBE using this growth approach [33]. They suggested that the growth process could have two stages. First, the epitaxial growth happens in the nano-pattern, which consists of initial nucleation, coalescence, and full coalescence. A single crystal is formed within each nanohole during this stage. At the second stage, the formed crystal undergoes morphology evolution, and changes into thermodynamically stable hexagonal shape. Consequently, the use of nano-patterned substrates provides a better control over the nanowire position, size, and aspect ratio. Shown in Figure 1.2, Bertness et al. reported their controlled wire diameter nanowires using silicon nitride masks [25]. Single-crystal nanowires with diameter from 500 $\mathrm{nm}$ to $1000 \mathrm{~nm}$ have been achieved using such selective areal growth approach. 


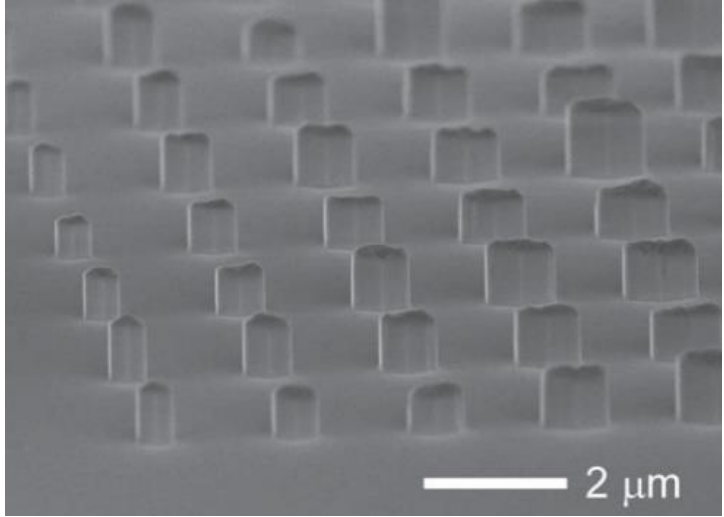

Fig. 1. 2: GaN nanowires with diameters from 500 to $1000 \mathrm{~nm}$ grown on patterned $\operatorname{SiN}_{x}$ substrate [25].

\subsection{Device fabrication of III-nitride nanowire LEDs}

Diverse geometries and arrangements of the nanowire structure can be obtained using various techniques. It requires that such nanostructured array can meet the demand in practical applications and avoid the electrical shortage during the deposition of the top $p$-type contacts [34-36]. One of solutions employs the spinon glass (SOG) to planarize the nanostructured array and isolate the nanowires. For example, Bai et al. reported the fabrication procedure of nanowire LEDs using a self-assembled Ni mask and SOG [3]. Figure 1.3(a) shows a SEM image of the formation of $\mathrm{Ni}$ islands annealed at $820{ }^{\circ} \mathrm{C}$ for 1 min under $\mathrm{N}_{2}$ ambient. In Figure 1.3(b), epi-wafer was etched down to the $n$-type layer to form the nanowire array structure using an ICP etching process. The SOG was then spin-coated on the nanowire sample followed by a curing process, as shown in Figure 1.3(c). An appropriate etch-back process needs to be performed to expose the $p$-type layer of the nanowires for later deposition of $p$-type metal contacts. Figure 1.3(d) shows the SOG coated nanowire LEDs after the etch-back process. In the next step, the mesa-etching and other procedures were conducted following standard fabrication of planar InGaN-quantum well based LEDs. Apart from SOG, similar gap-filling materials with properties of electrical isolation and high transparency, such as polyimide and SU8,
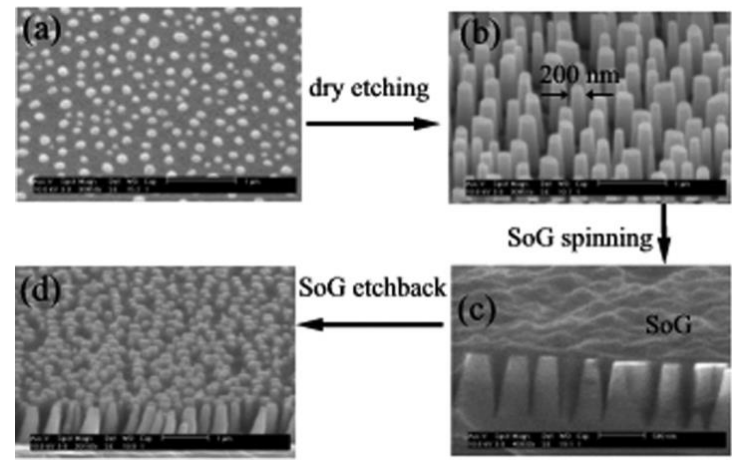

Fig. 1.3: SEM images of (a) self-assembled Ni masks; (b) GaN nanowires after ICP etching; (c) SOG was used to fill gaps between nanowires: (d) after the etching back process to expose the top $p$-type layer [3].

were also employed. Waag et al. reported another method to process the GaN nanowire LED by flip-chip packaging technology and bonding the GaN nanowire LED with silver nanoparticles or metal foils [37]. Chen et al. demonstrated the fabrication of nanowire LEDs by using chemical mechanical polishing for uniform nanowire contact and using $\mathrm{SiO}_{2}$ layer for sidewall passivation [38]. Another fabrication technique of nanowire LEDs is based on coalesced $p$-GaN growth, firstly reported by Kikuchi et al. [39]. By adjusting the growth parameters during $p$-GaN growth, the $p$-GaN part forms a complete layer allowing conventional planar device to be processed.

Philip et al. used polyimide as filling material in their nanowire LED fabrication since this material has low absorption of visible light and can be heated up to relatively high temperature [40]. The fabrication process starts with the epitaxial growth of nanowire LED structure, shown in Figure 1.4(a). Then, the nanowires are planarized by spin-coating of polyimide resist, illustrated in Figure 1.4(b), before applying $\mathrm{O}_{2}$ dry etching to expose the top portions of the nanowires. The metal contact layers include 5 $\mathrm{nm} \mathrm{Ni} / 5 \mathrm{~nm} \mathrm{Au} / 150 \mathrm{~nm}$ (ITO) are then deposited to form the top contacts, shown in Figure 1.4(c). The metal-grid of $10 \mathrm{~nm} \mathrm{Ni} / 100 \mathrm{~nm}$ Au layers are deposited on top of ITO to form contacts on $p$-type. Finally, $10 \mathrm{~nm} \mathrm{Ti} / 100 \mathrm{~nm}$ $\mathrm{Au}$ layers were then evaporated on the backside 
of the Si substrate for $n$-type contact, shown in Figure 1.4(d).

\section{Characteristics of III-nitride nanowire LEDs}

Various research groups demonstrated nanowirebased LEDs via top-down approach. Electroluminescence (EL) intensity was recorded for top-down based nanowire LED higher than that of the as-grown LEDs. Bai et al. reported that light output power enhanced by a factor of 1.35 and 1.83 , for the blue and green nanowire LED compared with the as-grown blue and green LEDs, respectively [3]. Their experiment was performed at room temperature and at current injection of $20 \mathrm{~mA}$, shown in Figure 2.1. More significant enhancement in the light output power for the green nanowire LED manifested that the emission enhancement is mainly attributed to the large strain relaxation occurring during the fabrication of nanowire and thus a significant enhancement in the internal quantum efficiency (IQE). Using the method of silica nanoparticle lithography, Wang et al. reported nanowire LEDs with nearly constant emission wavelengths [35]. Chang et al. investigated the light output of nanowire LED varied with different rod etching depths [41]. They found that the shallower nanowires possess the fewer surface defects and thus give a higher light output power, while longer nanowires show smaller efficiency droop due to more relaxed strain. Chen et al. investigated the efficiency droop of nanowirebased LED structure with different strains with different choice of nanowire sidewall passivation materials [42]. They reported that Auger recombination is significant at low injection currents, while increase number of leakage carriers causes efficiency drop at high injection currents. Ke et al. presented that a narrower radiation profile was demonstrated from the nanowire LED [43].

In case of bottom-up nanowire LEDs, significantly progress was reported. As shown in Figure 2.2(a), Kikuchi et al. reported that strong EL emissions at green $(530 \mathrm{~nm})$, yellow (580 $\mathrm{nm})$, and red $(645 \mathrm{~nm})$ were achieved at room temperature [39]. This was mainly attributed to the high electron-hole recombination efficiency, achieved from the quantum disks in nanowires. Reported by Kim et al., strong EL emission at $\sim 460 \mathrm{~nm}$ is observed for a well-in-a-wire LED with negligible peak wavelength shift with the increase of injection current as shown in Figure 2.2(b) [44]. An enhancement factor of more than 4.3 times was measured for the light output power of such well-in-a-wire LEDs compared to conventional LEDs at $20 \mathrm{~mA}$, illustrated in Figure 2.2(c) [44]. Due to the enhanced carrier confinement and self-surface passivation, the dot-in-a-wire reported by Nguyen et al. showed promising device performance for future solidstate lighting and display applications [36]. Additionally, the color properties of those dot-in-awire LEDs could be controlled by adjusting the compositions and/or sizes of the active region via engineering the growth conditions. With the use of such dot-in-a-wire structures, full-color nanowire LEDs were demonstrated. By mixing these emission colors in a single nanowire, Nguyen et al. achieved truly white light emission covering from $\sim 430 \mathrm{~nm}$ to $>750 \mathrm{~nm}$ which is nearly the entire visible spectrum. High brightness emission from the white LED is also illustrated in the inset of Figure 2.3(a). A consistent blue shift (up to $\sim 8.4 \mathrm{~nm}$ ) was observed when the injection current was varied from 30 to 500 $\mathrm{mA}$. The IQE of the nanowire LEDs were then measured by comparing the EL intensity at 300 $\mathrm{K}$ to that measured at $5 \mathrm{~K}$. The devices exhibit an IQE of $\sim 36.7 \%$ at room temperature under electrical injection and efficiency droop was not observed up to injection current density of 200 $\mathrm{A} / \mathrm{cm}^{2}$ [50], shown in the Figure 2.3(b).

Although compare with the conventional planar LEDs, GaN nanowire LEDs offer many potential advantages such as significantly reduced dislocation densities and polarization fields [36, 45-47], as well as enhanced light extraction efficiency (LEE) [48], however, III-nitride nanowire based LEDs generally exhibit slow rise of EQEs with increased injection current and extremely low output power. For example, the peak quantum efficiency of such devices can only be measured under relatively large injection conditions $\left(>100 \mathrm{Acm}^{-2}\right)[6,8,9]$, compared to that $\left(\sim 10-20 \mathrm{Acm}^{-2}\right)$ of InGaN/GaN planar 


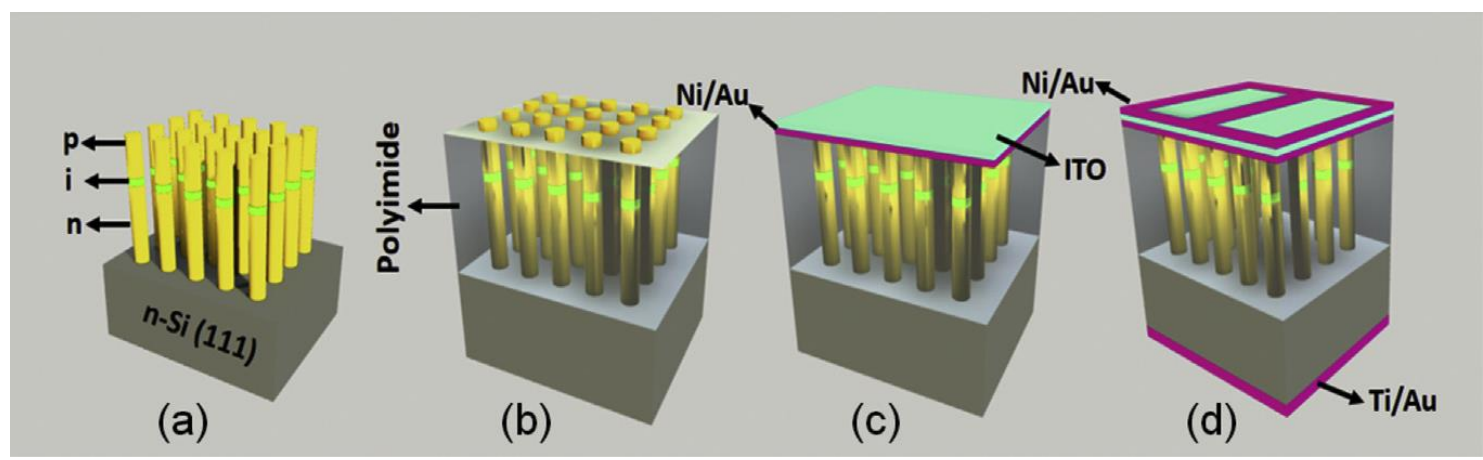

Fig. 1. 4: The fabrication procedure of nanowire LEDs on a silicon substrate [40].

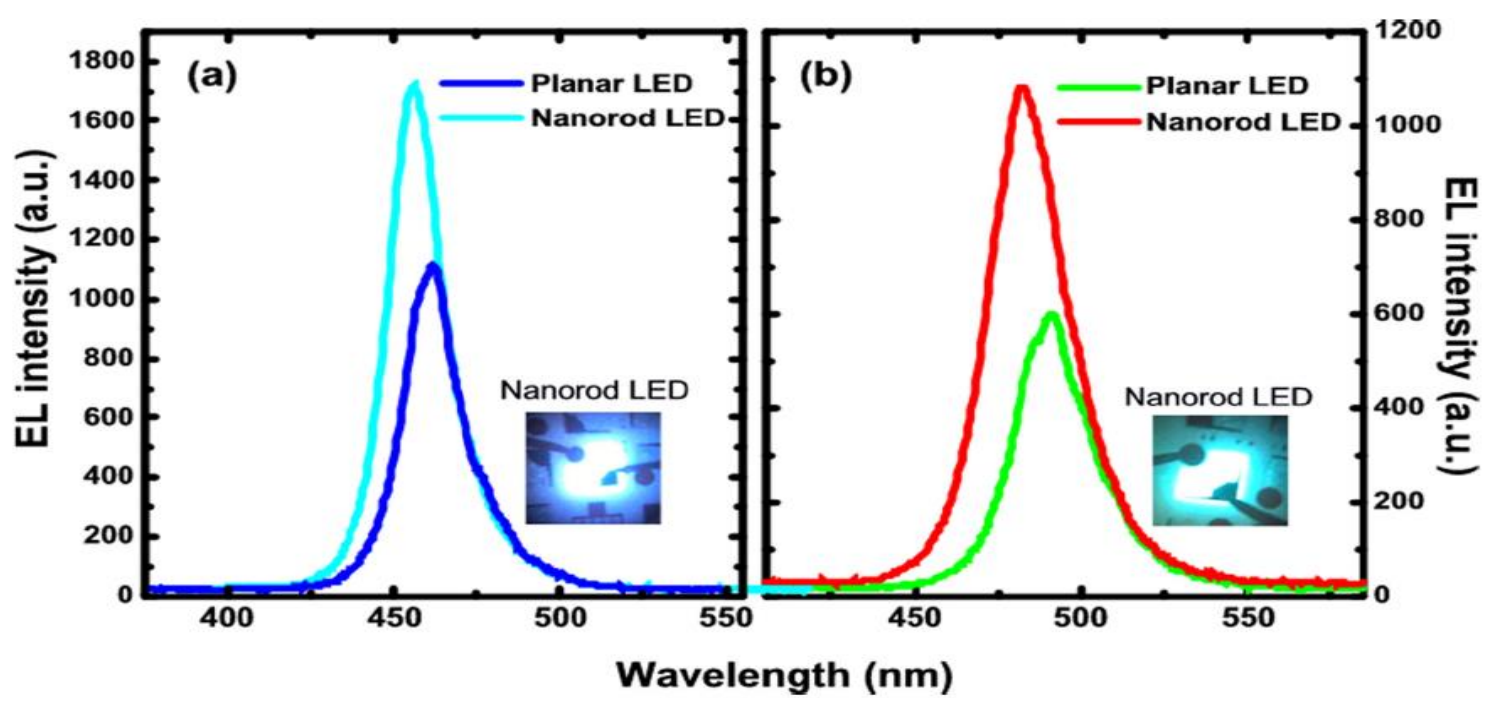

Fig. 2. 1: Room temperature EL spectra of LEDs measured at $20 \mathrm{~mA}$ injection current for blue (a) and (b) green nanowire LEDs. The insets show photographic images of the emissions at $1 \mathrm{~mA}$ current [3].

LEDs [10-12], indicating the presence of a significant level of nonradiative carrier recombination processes [13]. The surface states/defects are identified as one of the main factors that strongly affect the performance of nanowire LEDs [49]. The performance of such nanoscale LEDs is more susceptible to electron leakage out of the device active region due to the presence of large densities of states/defects along the wire surface and the one-dimensional carrier transport process. The resulting carrier loss and nonradiative carrier recombination on nanowire surfaces severely limit the device performance. The next session will present the main underlying causes of the poor carrier injection efficiency and strategies to minimize the carrier loss processes for the enhanced performance of nanowire LEDs.

\section{Carrier dynamics and loss mechanism of axial nanowire LEDs}

In this section, plausible causes of carrier losses in axial nanowire LEDs will be discussed. The probable carrier loss processes can be listed as Auger recombination, electron overflow/leakage, and nonradiative surface recombination. To analyze the IQE of nitride-based LEDs, the ABF model $[36,51]$ is utilized which is described be- 

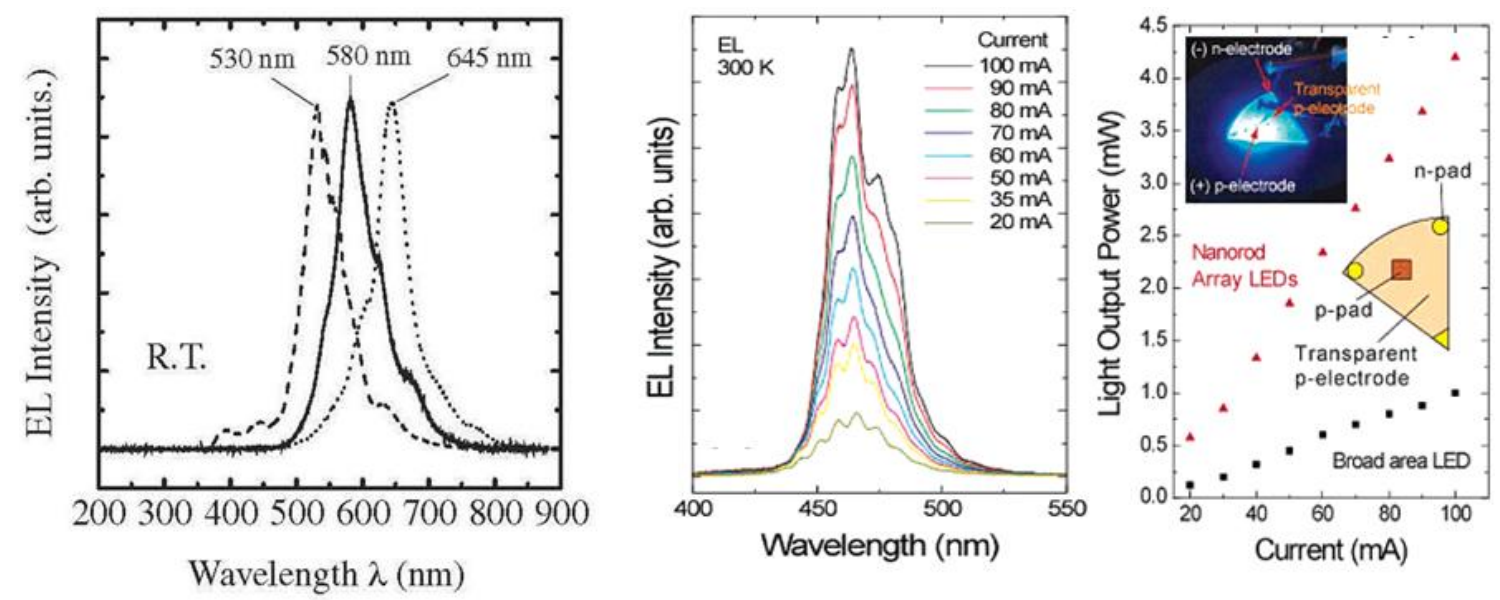

Fig. 2.2: (a) EL spectra of disk-in-a-wire with emission peaks at $530 \mathrm{~nm}, 580 \mathrm{~nm}$, and $645 \mathrm{~nm}$, respectively [39]. (b) Room temperature EL of well-in-a-wire LED with varying injection currents [44]. (c) Light output power of well-in-a-wire LEDs and thin film LEDs versus injection currents [44].

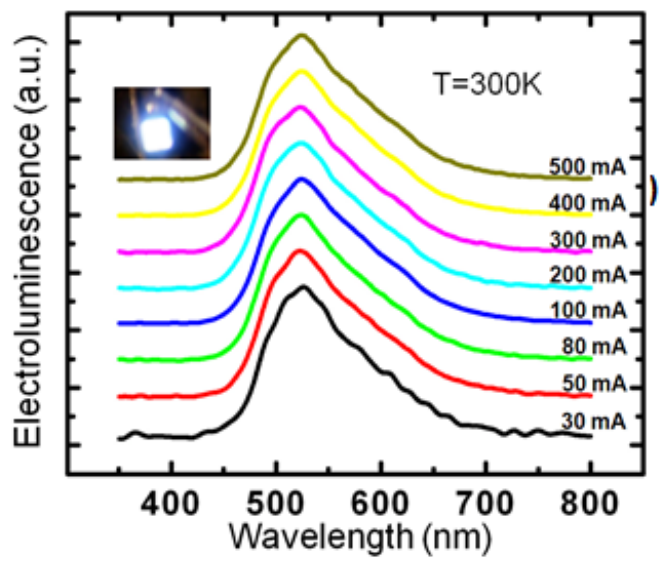

(a)

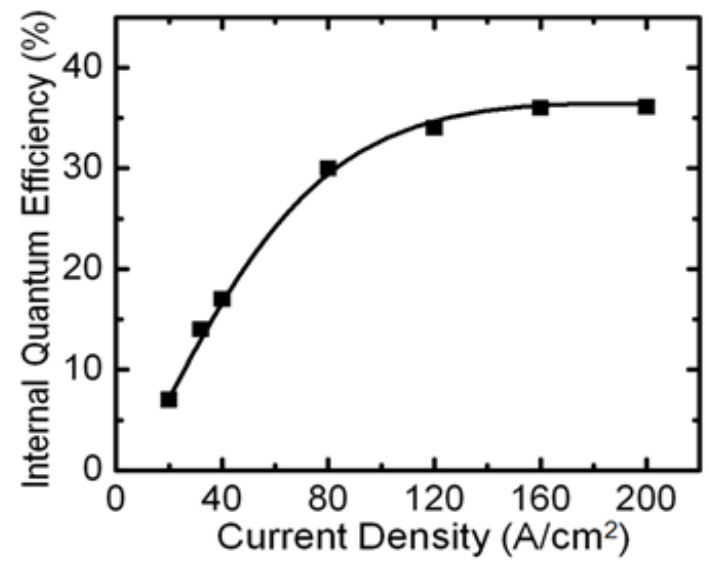

(b)

Fig. 2.3: (a) EL spectra of phosphor-free white LED under different injection current levels and the optical image of white emission is shown in the inset. (b) IQE of phosphor-free dot-in-a-wire nanowire white LED measured under electrical injection condition [50].

low:

$$
\eta_{i}=\frac{B N^{2}}{A N+B N^{2}+f(N)}
$$

where $\eta_{i}$ is presented for the device IQE, and $\mathrm{N}$, $\mathrm{A}$, and $\mathrm{B}$ are the carrier density in the active region, the $\mathrm{SRH}$ nonradiative recombination coefficient, and the radiative recombination coefficient, respectively. $f(N)$ represents the third or other higher order effects.

The performance of nanowire LEDs is defined by their external quantum efficiency (EQE), which is a product of carrier injection efficiency,
IQE and LEE. The performance of such devices has been severely limited by their low IQE and low LEE. The Auger recombination, poor hole transport, electron leakage and overflow [52, 53], and defects/dislocations are among the loss mechanism which can reduce the IQE in the electrically injected white-color nanowire LEDs.

\subsection{Auger recombination}

Auger recombination, which refers to a threecarrier nonradiative recombination process, is 
found to be a reason for the performance degradation in GaN based LEDs at higher carrier concentrations [54-56]. In this process, a third carrier gets the excess energy released by the recombination of an electron-hole pair. The third carrier, which can be an electron or a hole with high kinetic energy, is then excited to a higher energy state, which leads to a large efficiency droop observed in GaN LEDs. Shen et al. measured the Auger recombination coefficient for InGaN/GaN quantum well LEDs in the range of $\sim 10^{-30}$. $10^{-31} \mathrm{~cm}^{6} \mathrm{~s}^{-1}$ [55]. Recent studies showed that the Auger recombination processes can be either direct or indirect [57]. While the direct Auger recombination is weak in the nitride based devices due to wide-bandgap materials, the indirect Auger recombination assisted by phonon and alloy scattering was suggested as the dominant factor causing the large Auger coefficient, which affects the efficiency of nitride based devices at high injection currents [57]. Zhang et al. directly measured the Auger coefficient values of $\mathrm{In}_{0.1} \mathrm{Ga}_{0.9} \mathrm{~N} / \mathrm{GaN}$ multi-quantum wells and confirmed that Auger recombination is largely responsible for efficiency droop at high injection currents [56]. Iveland et al. further concluded that Auger recombination can explain the observed efficiency droop in $\mathrm{InGaN} / \mathrm{GaN}$ quantum well LEDs [58].

On the other hand, in case of top-down nanowire structure, Renwick et al. found that Huang-Rhys factor of nanowires is much smaller than that of as-grown multi-quantum well sample due to a reduced coupling between LOphonon and exciton [59]. It is suggested that Auger recombination is significantly reduced due to the strain relaxation during the fabrication of nanowires. For bottom-up nanowires, Guo et al. measured the Auger recombination coefficients in InGaN nanowires and $\mathrm{InGaN} / \mathrm{GaN}$ dot-in-wire samples by performing excitation dependent PL and time resolved PL measurements and reported the Auger recombination coefficient for their nearly defect free $\mathrm{InGaN} / \mathrm{GaN}$ nanowires with a much smaller value of $\sim 10^{-32}$ to $\sim 10^{-33} \mathrm{~cm}^{6} \mathrm{~s}^{-1}[60]$. Nguyen et al. also performed a detailed measurement of the temperature dependent emission characteristics of dotin-wire white LEDs and measured the Auger recombination coefficient of $\sim 10^{-34} \mathrm{~cm}^{6} \mathrm{~s}^{-1}$ or less
[61]. Their studies strongly suggest that Auger recombination plays a very small, or negligible role on the performance of nanowire LEDs due to the drastically reduced defect densities and improved crystal quality in nanowire structures.

\subsection{Non-uniform carrier distribution}

Sapphire, $\mathrm{SiC}$ and $\mathrm{Si}$ are among the most commonly used substrates for nitride-based LEDs. These materials have different lattice constants which result in large lattice mismatches. The polarization field caused by the large lattice mismatch plays an important role in degrading the performance of nitride-based LEDs. The polarization field, including the spontaneous and strained-induced piezoelectric polarization, spatially separates the electron and hole within the quantum wells. This is known as QCSE $[62,63]$. The spatial separation of carriers decreases the radiative recombination and greatly degrades the performance of LEDs. Figure 3.1 shows the band diagram of a single quantum well suffering the effects of polarization. The electron and hole wave functions are separated and moved toward different directions. Compared to the symmetric wave function without polarization field, the asymmetric wave function of electron and hole reduces the overlap of confined electrons and holes, consequently resulting in a low radiative recombination rate.

The device performance is highly affected by the heavy effective mass and low mobility of holes which cause highly inefficient hole transport in device active regions $[64,65]$. Compared to uniform distribution of electrons, the holes are generally distributed in the quantum wells near the $p$-doped GaN layer, and are significantly degraded toward the $n$-GaN region $[36$, 66, 67]. This enhances the Auger recombination significantly and leads to increase the electron overflow and nonradiative surface recombination at high current injections, which further limit the light output power. The barrier thickness of the quantum well active region can be optimized to improve the hole distribution along the active region [68]. Utilizing a thinner barrier near the $p$-type GaN layer was studied 
by Tsai et al. which leads to increase the hole injection, therefore reducing the electron leakage [69]. Nguyen et al. reported that, the hole transport to the nanowire LED active region could be improved by applying $p$-type modulation doping approach [36]. The hole population is greatly increased, resulting in the more uniform carrier distribution across the device active region which significantly improved the electrical performance of nanowire devices. As such, the $p$-doped nanowire LED exhibits high IQE of $\sim 57 \%$, while the undoped LED has much lower IQE of $\sim 37 \%$.

\subsection{Electron leakage}

In addition to the previously described carrier loss mechanism, the electron leakage and overflow is another major process which can highly affect the performance of nanowire LEDs at high current levels [70-75]. As mentioned before, the performance of GaN LEDs is adversely affected by the poor hole injection and transportation caused by the heavy effective mass, and poor mobility of holes [66]. The highly nonuniform distribution of carriers results in a significantly enhanced electron overflow, and efficiency droop at high injection levels. The presence of the polarization field could tilt the bandedge, resulting in the separation of electrons and holes. Therefore, carrier recombination is also
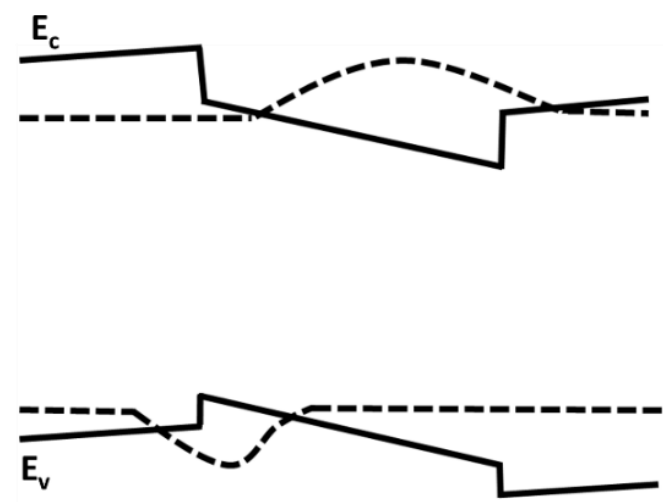

Fig. 3.1: The conduction and valence band edges of a single QW including the effects of polarization. The electron and hole wave functions shown in the figure are shifted to the right and left, respectively. reduced. Polarization field can also effectively diminish the barrier height for electrons, which enhances the electron leakage out of the LED active region. The overflowed electrons can recombine with the holes in the $p$-GaN region before they can reach the active region, thus degrades the LED performance by reducing the radiative recombination in the active region. Even though nanowire LEDs exhibit reduced polarization field, the effect of electron overflow/carrier leakage may become more severe, due to the presence of large densities of states/defects along the wire surface and the one-dimensional carrier transport process.

Figure 3.2 shows the electron overflow mechanism in the LED structure. The electrons and holes injected into the device can recombine in the active region. However, some electrons having sufficient energy can surmount the barrier and flow out of the active region without recombining with holes. The electron overflow has been reduced by implementing an Electron Blocking Layer (EBL) in the $p$-side of the LED. A wide bandgap energy AlGaN layer can be typically inserted between the active region and the $p$-side in the GaN LEDs to prevent the electrons from overflowing out of the active region. However, the EBL could reduce the hole injection efficiency depending on the band offset.

Nguyen et al. addressed the presence of electron overflow and performed a detailed study of electron overflow on the external quantum efficiency (EQE) of InGaN/GaN dot-in-a-wire

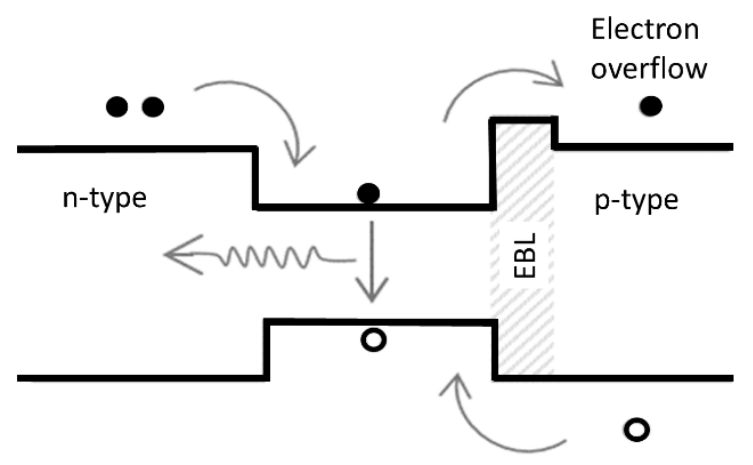

Fig. 3. 2: The electron overflow mechanism in the LED structure which shows overflowing some electrons with sufficient energy out of the active region without recombining with holes. 

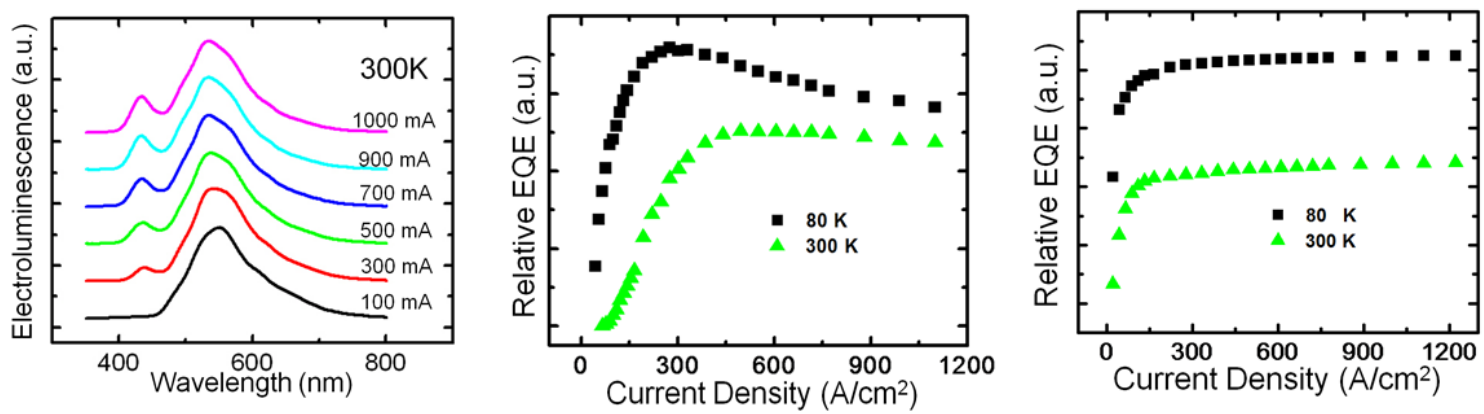

Fig. 3.3: (a) Normalized EL spectra of a nanowire LED with a test quantum well under various injection currents; (b) Relative EQE with injection current for the emission from the active region and the test well measured at room temperature. (c) Relative EQE versus injection current for emission from the InGaN/GaN nanowire LED with the incorporation of AlGaN EBL layer [51].

LEDs under different temperatures by incorporating a $p$-doped InGaN/GaN test quantum well between the active region and $p$-GaN segment [51]. In such structure, electrons overflow from the active region can recombine with holes in the InGaN/GaN quantum well, which has larger bandgap energy than that of the active region. Shown in Figure 3.3(a), only one peak emission at $\sim 550 \mathrm{~nm}$ which is from the quantum dot active region can be observed at low injection current levels $(<100 \mathrm{~mA})$. However, due to the enhanced electron overflow, emission from the test well $(\sim 430 \mathrm{~nm})$ becomes progressively stronger with increasing current. Therefore, at high injection current levels, electron overflow in the nanowire LED devices is more pronounced.

Additionally, efficiency droop was observed in nanowire LEDs and become more severe at reduced temperatures due to the increased electron overflow, as shown in Figure 3.3(b). It is suggested that efficiency droop is mostly caused by electron overflow, rather than Auger recombination in nanowire LEDs, since Auger recombination rate is typically reduced at low temperatures. Nguyen et al. showed that electron overflow could be largely suppressed with the use of a $p$-doped AlGaN electron blocking layer in nanowire structures [51]. They observed no efficiency droop for injection currents of $\sim 1200 \mathrm{~A} / \mathrm{cm}^{2}$ at both room temperature and $80 \mathrm{~K}$ for $\mathrm{In} \mathrm{GaN} / \mathrm{GaN}$ dot-in-a-wire LED with the incorporation of $\mathrm{AlGaN}$ electron blocking layer, shown in Figure 3.3(c). Djavid et al. proposed the use of tunnel injection to reduce electron overflow in nanowire LEDs [76].

\subsection{Nonradiative surface recombination}

The defect states in the bandgap act as Shockley-Read-Hall (SRH) non-radiative recombination centers, which result in severe degradation in the performance of GaN LEDs. The origin of defect states can be related to the presence of dislocations, which are commonly caused by large lattice mismatches between the nitride-based materials and the substrates such as sapphire, $\mathrm{SiC}$ and $\mathrm{Si}$. The dislocations are generated in the layers grown epitaxially on the substrate due to the lattice mismatch, as the thicknesses of layers are above a critical thickness. The dislocations result in defect states in the bandgap and consequently severe degradation in the performance of the GaN LEDs [7779]. Therefore, it is crucial to reduce the lattice mismatch of LEDs and minimize the performance degradation in the optical and electrical performance of these devices. The defect density is higher in the planar GaN LEDs due to the lattice mismatch of epitaxial layer with respect to substrates.

The nanowire structure, having high surfaceto-volume ratio, offers more area for the photons to escape. However, it also causes large surface states. The defect states are introduced in the forbidden bandgap of the semiconductor due to the termination of the crystal structure periodicity at the surface. The performance of a nanowire is limited by the surface states, which can reduce the carrier injection efficiency by capturing carriers from the active region [41]. 

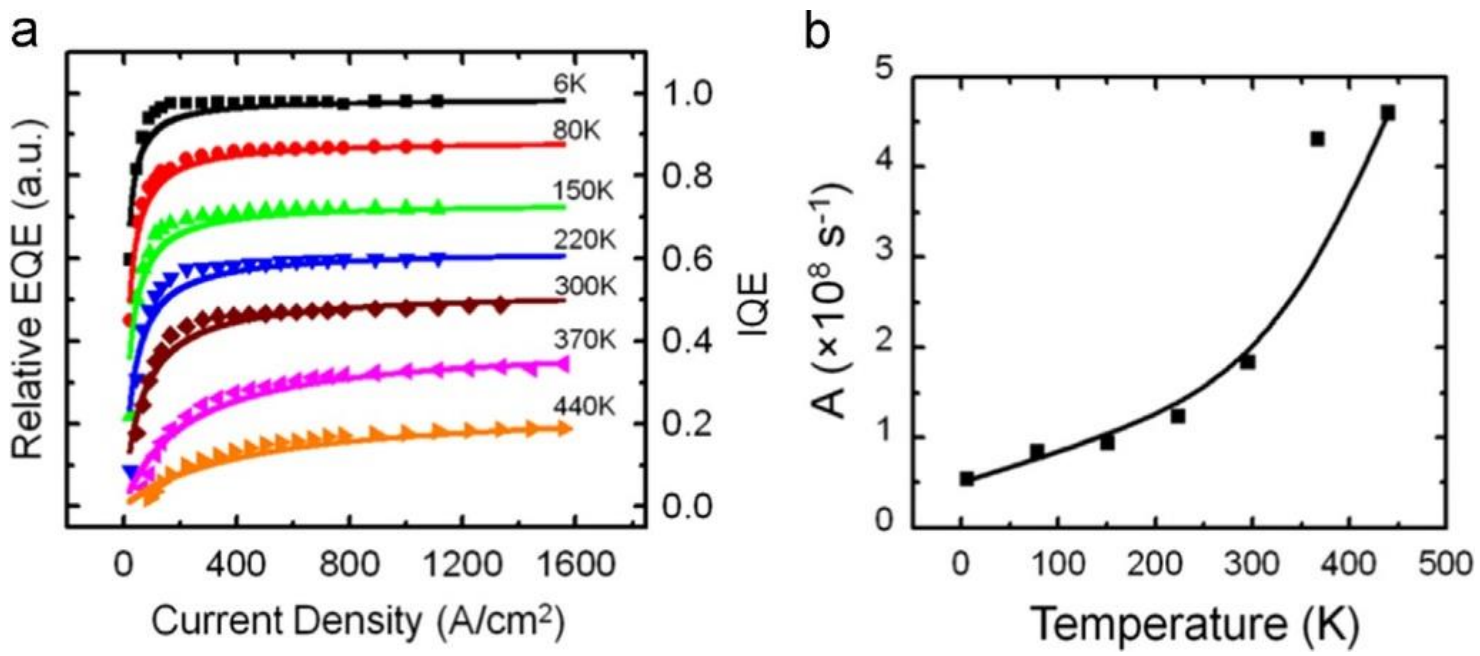

Fig. 3.4: Temperature dependent EQE vs. injection current densities of the InGaN/GaN dot-in-a-wire LED measured from $6 \mathrm{~K}$ to $440 \mathrm{~K}$. The simulated internal quantum efficiency curves at each temperature are shown as solid curves. (b) Variation of the SHR nonradiative recombination coefficient (A) measured at different temperatures [61].

Generally, nanowire LEDs exhibit peak quantum efficiency at relatively high injection levels which is $>100 \mathrm{~A} / \mathrm{cm}^{2}[61,80]$. This value is significantly higher than that of conventional InGaN/GaN quantum well blue LEDs, generally in the range of $\sim 10-20 \mathrm{~A} / \mathrm{cm}^{2}[80,81]$. Nguyen et al. performed detailed analysis of the temperature-dependent emission characteristics of $\mathrm{InGaN} / \mathrm{GaN}$ nanowire LEDs, and showed that the EQE of nanowire LEDs shows a slow rising trend under low current injections, while the peak quantum efficiency occurs at relatively high injection current of $\sim 250 \mathrm{~A} / \mathrm{cm}^{2}$ at $300 \mathrm{~K}$ as shown in Figure 3.4(a) [61]. The slow rise trend of EQE is directly related to the large SRH nonradiative recombination rate which is explained following the ABF model analysis. With the increased temperature, the $\mathrm{SRH}$ recombination becomes progressively increased. Figure 3.4(b) shows the derived SRH coefficients (A) of InGaN/GaN nanowire LEDs versus temperature. The SRH coefficient $(\mathrm{A})$ varied from $\sim 5.5$ $\times 10^{7} \mathrm{~s}^{-1}$ to $4.6 \times 10^{8} \mathrm{~s}^{-1}$ for different temperature from 6 to $440 \mathrm{~K}$ [61]. Compared to conventional InGaN/GaN quantum well LEDs, due to the large nonradiative surface recombination, the InGaN/GaN nanowire LEDs shows much higher A coefficient at room temperature $\left(\sim 10^{7} \mathrm{~s}^{-1}\right)$ [82]. The large nonradiative surface recombination also results in efficiency droop under high injection currents because of leakage effect [81, 83]. Zhang et al. reported that the PL intensity of top-down etched nanowire LEDs exhibited efficiency droop with increasing excitation power. The efficiency droop was dramatically increased with decreasing temperature. They suggested that the strong nonradiative recombination on the nanowire surface is the main reason causing efficiency droop. Using different passivation techniques, the nonradiative surface recombination can be effectively reduced and consequently the performance of nanowire LEDs can be significantly improved [84-87]. Several materials have been reported for the surface passivation of III-V semiconductors including $\mathrm{SiO}_{2}, \mathrm{SiN}_{x}$ and sulfide [88-90]. The sulfur surface passivation technique was effectively used to remove the surface states and recovering the band-edge emission of InAs nanowires [15].

\subsection{Carrier injection efficiency of nanowire LEDs}

The carrier injection efficiency in nanowire devices can be extremely small ( $10 \%)$ compared to the conventional quantum well LEDs with near-unity carrier injection efficiency. This can 
be explained by the dominance of nonradiative surface recombination. The electron concentration in the device active region of nanowire LEDs can be 10 times smaller than that of thinfilm LEDs at the same current injection conditions [85]. Consequently, the carrier lifetime for nanowire LEDs is measured very small ( $\sim 0.2 \mathrm{ns,}$ or less) [91-93]. The carrier injection efficiency is calculated by Zhang et al. for different surface recombination velocities and nanowire diameters as shown in Figure 3.5 [94]. The carrier injection efficiency decreases significantly with decreasing nanowire diameter, and it is well below $10 \%$ for nanowire diameters in the range of $100 \mathrm{~nm}$. Such an extremely small carrier injection efficiency results in a very low output power which is similar to conventional nanowire LEDs.

The carrier injection efficiency for relatively small nanowires is significantly smaller than large nanowires. Compared to other III-V materials, although the surface recombination velocity is smaller for GaN material [95], the surface states defects can significantly reduce the nanowire LEDs performances with the large surface-to-volume ratios. The large surface-tovolume ratio is necessary to have effective lateral stress relaxation of nanowire LEDs and enhance the large LEE. Reducing the surface recombination velocity is the most effective way for the

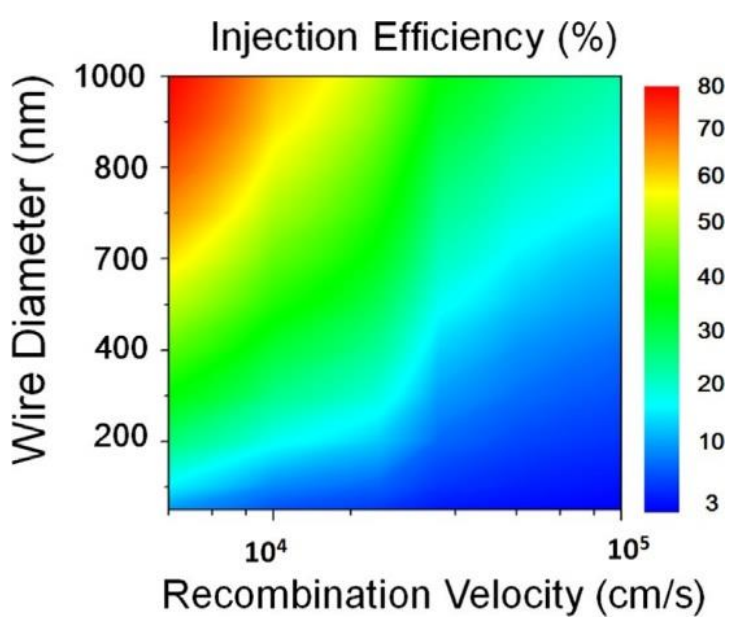

Fig. 3.5: Variations of the carrier injection efficiency of the nanowire LEDs vs. surface recombination velocities (from $5 \times 10^{3} \mathrm{~cm} / \mathrm{s}$ to $1 \times 10^{5}$ $\mathrm{cm} / \mathrm{s}$ ) for different nanowire diameters (from $50 \mathrm{~nm}$ to $1000 \mathrm{~nm}$ ) [94]. carrier injection efficiency enhancement, which was proposed by utilizing the AlGaN core -shell around the nanowire LED structures [96, 97].

\section{Typical designs for the enhanced performance of III-nitride nanowire LEDs}

In this section, different III-nitride nanowire LED designs such as heterostructure, well/disk/dot-in-a-wire, polarized induced, tunnel junction induced nanowire LEDs will be discussed.

\subsection{Heterostructure nanowire LEDs}

Hahn et al. reported that $\operatorname{In}_{x} \mathrm{Ga}_{1-x} \mathrm{~N}$ nanowire LEDs with In composition varies from 0.06 0.43 , were grown on sapphire substrate by using halide chemical vapor deposition (HCVD) method [98]. Shown in Figure 4.1(a), LED devices are formed on $p$-GaN grown on sapphire substrate. The emission wavelength of devices can be tuned by controlling the In composition in nanowires, as shown optical images and emission spectra in Figures 4.1(b) and (c), respectively. Figure 4.1(d) presents the current dependent electroluminescence of nanowire LED devices with indium composition of about 0.28 .

Full-color emissions were demonstrated with an IQE in the range of $20-35 \%$ by using $\mathrm{InGaN} /-$ GaN double heterostructures nanowire structure, reported by Guo et al. [99]. Figure 4.2(a) shows the TEM image of a single InGaN nanowire showing the variation of indium composition along the InGaN wire. No defects were observed at the interface of $n$-GaN/InGaN as illustrated in the inset of Figure 4.2(a). Moreover, the peak emission wavelength can be controlled in the entire visible wavelength range by changing the In composition in the LED active region, illustrated in Figure 4.2(b). Additionally, white light emission was also obtained using this approach as shown in the inset of Figure 4.2(b). 
a
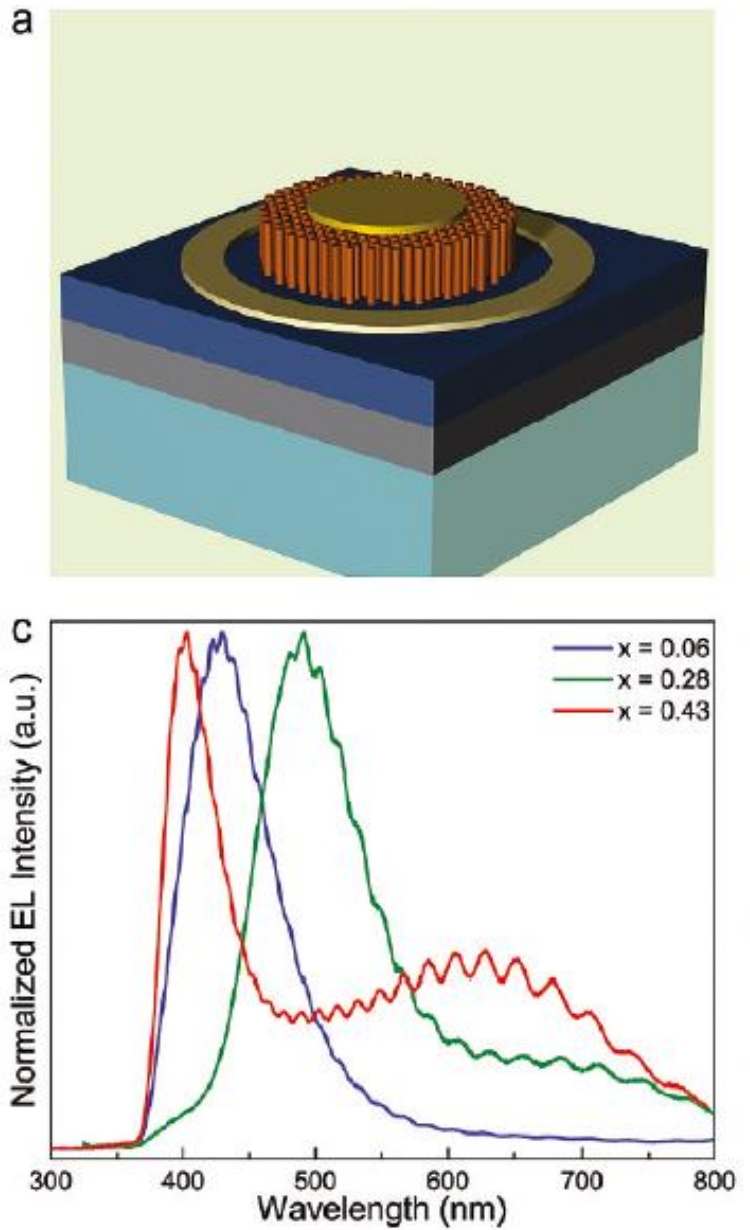

b
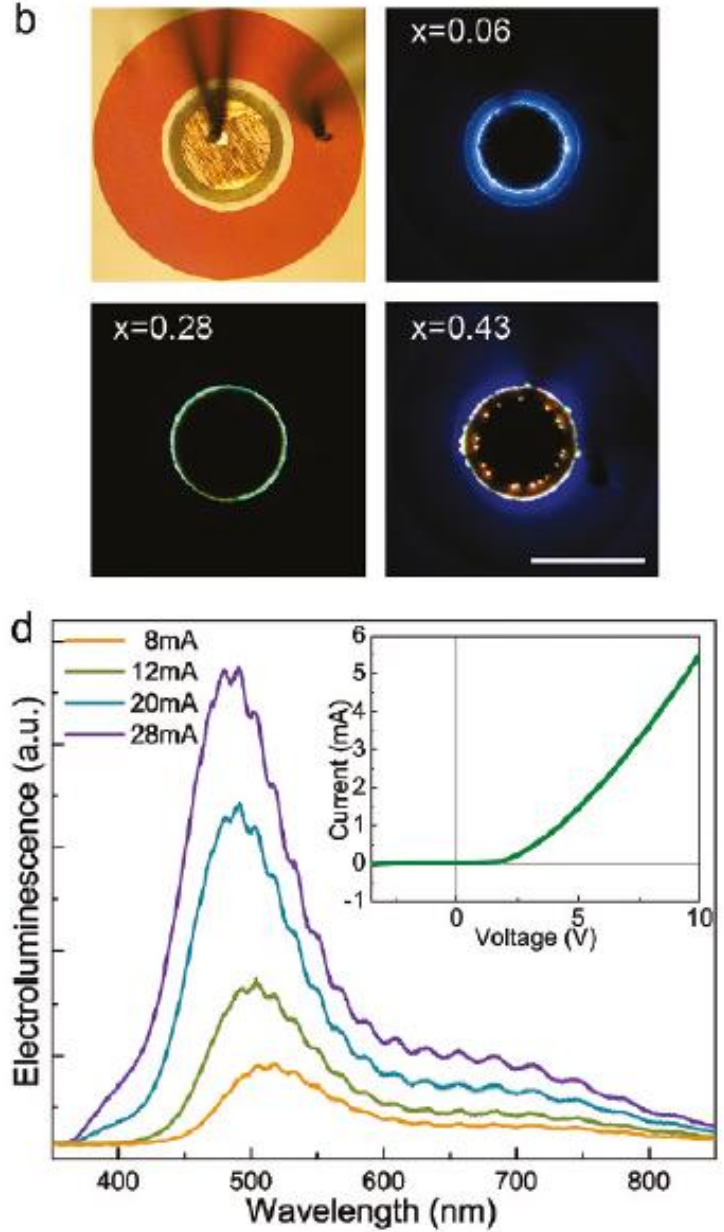

Fig. 4.1: (a) Schematic illustration of a fabricated InGaN LED device. (b) Top-view images of the LED devices of different In compositions. (c) Normalized EL spectra of LED devices. (d) Electroluminescence under different injection currents for the LED device with In composition of 0.28 [98].

\subsection{Disk/Well-in-a-wire LEDs}

To increase the carrier confinement in the LED active region, well/disk/dot-in-a-wire LED structures were developed in which multiple In$\mathrm{GaN} / \mathrm{GaN}$ wells, disks or dots were embedded in GaN wires. Kikuchi et al. [39] demonstrated an efficient approach to enhance the carrier confinement by the use of InGaN/GaN multiple quantum disks (MQD) embedded in a single nanowire by MBE on Si (111). Figure 4.3 shows the crosssectional schematic diagram and SEM image of InGaN/GaN MQD nanowire LEDs. Moreover, the top segment $p$-GaN of the nanowires was laterally grown to form coalesced film to facilitate the device fabrication. The strong emis- sions at green $(530 \mathrm{~nm})$, yellow $(580 \mathrm{~nm})$, and red $(645 \mathrm{~nm})$ were achieved due to enhanced carrier confinement in the LED active region. Kim et al. reported multiple quantum well (MQW) nanorod arrays grown by metal organic-hydride vapor phase epitaxy (MO-HVPE) on sapphire substrate. The corresponding SEM and TEM images are shown in Figure 4.4 [44]. Detailed measurements indicated that the output light from such MQW nanorod LEDs was about 4.3 times stronger than that of conventional LEDs. Armitage et al. also reported that multi-color emission from single InGaN quantum well grown over GaN nanowire by MBE [100]. 

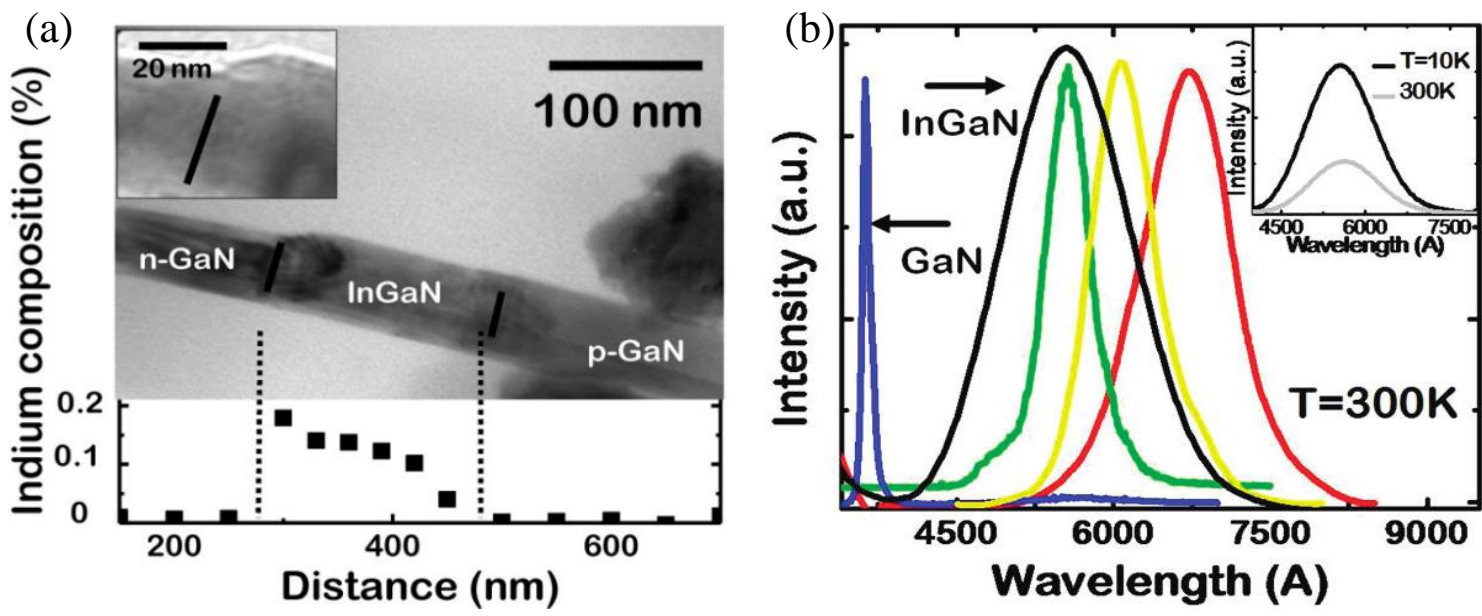

Fig. 4. 2: (a) STEM image of an InGaN/GaN double heterostructure single nanowire and the EDSX profile of indium composition along the entire length. No defects are recorded at the $n$-GaN/InGaN interface, as shown in the inset (b) Room-temperature PL spectra of In GaN nanowires with different In contents and the PL spectra of a white emission LED measured at room temperature and $10 \mathrm{~K}$ [99].

(a)

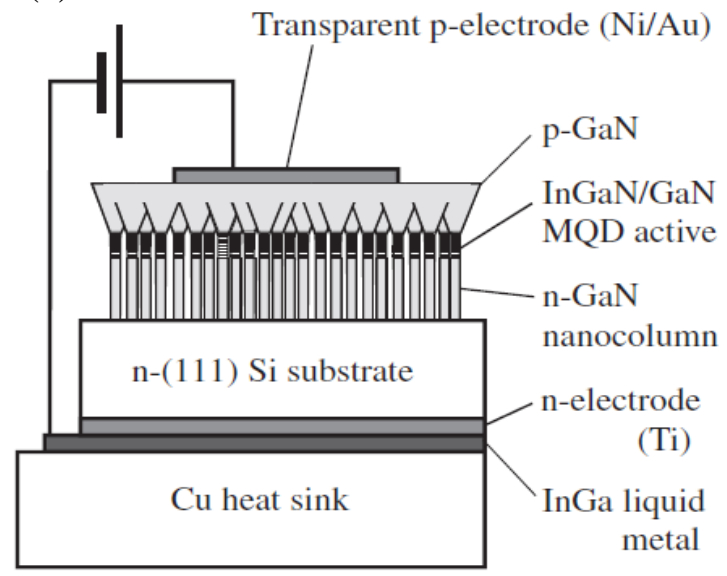

(b)

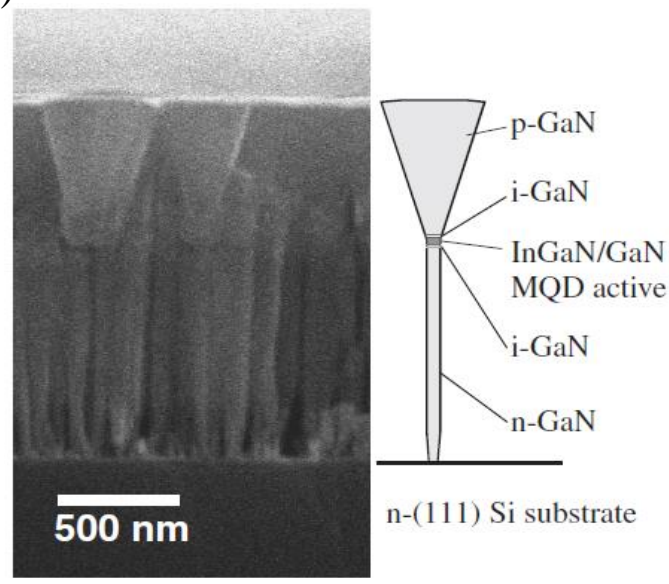

Fig. 4. 3: (a) Cross-sectional schematic diagram (b) SEM image of InGaN/GaN multiple quantum disk nanocolumn LED and layer by layer schematic of a single nanocolumn structure [39].

They found that the emission spectra from nanowires are varied with different diameters and densities. Accordingly, the phosphor-free white LEDs have been demonstrated by optimizing both the GaN wire density and InGaN growth conditions. Guo et al. [80] demonstrated a high performance InGaN/GaN ten disk-in-awire LED with no efficiency droop up to high injection current of $400 \mathrm{~A} / \mathrm{cm}^{2}$. The SEM image and TEM image of InGaN/GaN disk-in-awire are shown in the Figure 4.5(a) and Figure 4.5(b). Information regarding quantum effi- ciency and EL intensity as a function of current injection is provided in the Figure 4.5(c).

\subsection{Dot-in-a-wire LEDs}

The performance of nanowire LED devices can be significantly improved by achieving superior carrier confined in three dimensions. Nguyen et al. recently reported an $\mathrm{InGaN} / \mathrm{GaN}$ dotin-a-wire heterostructure grown by MBE [36]. In their LED structures, multiple $\mathrm{InGaN} /-$ 

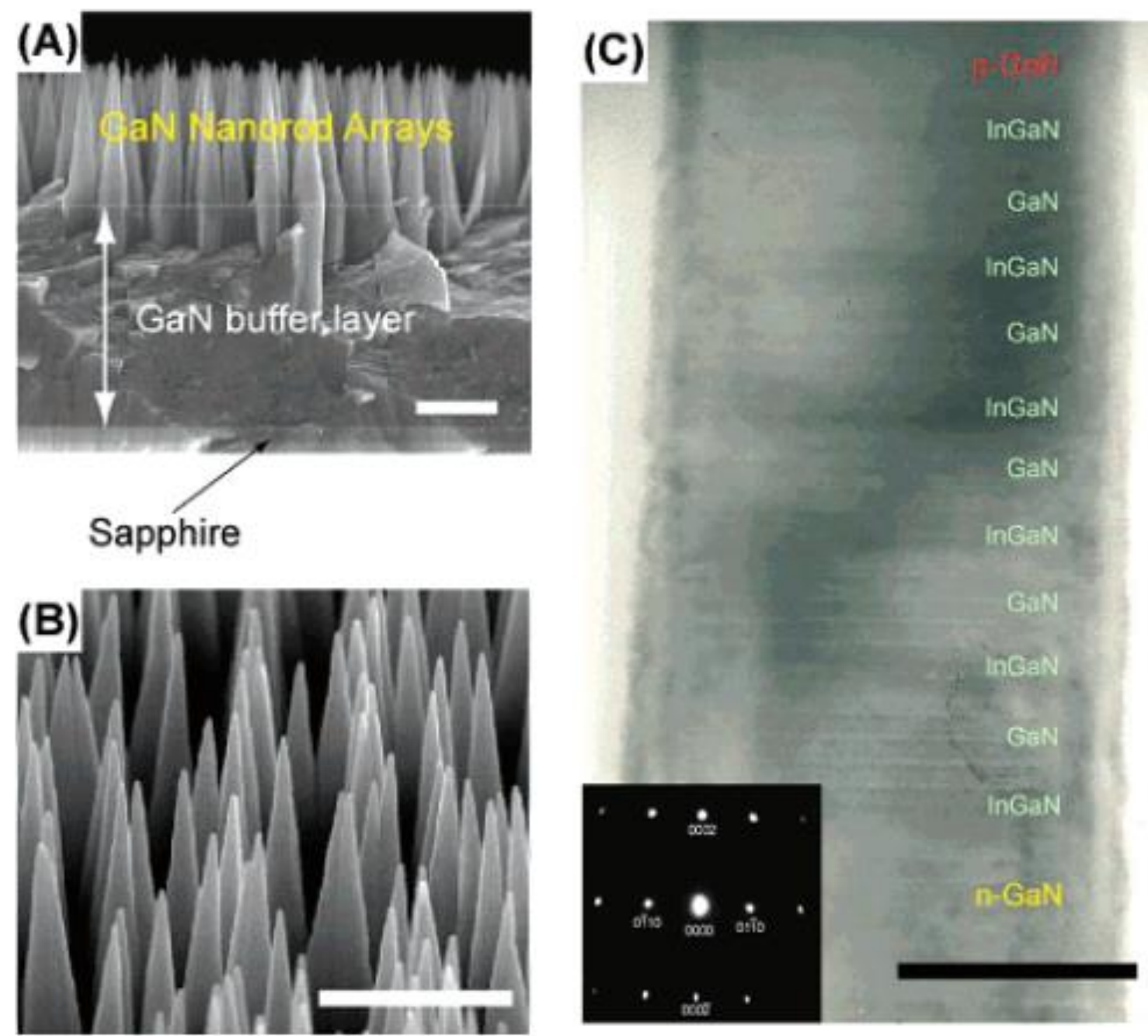

Fig. 4.4: (a) Cross-sectional view SEM of InGaN/GaN MQW nanorod arrays with $500 \mathrm{~nm}$ scale bar, (b) $30^{0}$ tilt view of SEM image of InGaN/GaN MQW nanorod arrays with $500 \mathrm{~nm}$ scale bar, (c) TEM image of InGaN/GaN MQWs with $30 \mathrm{~nm}$ scale bar [44].
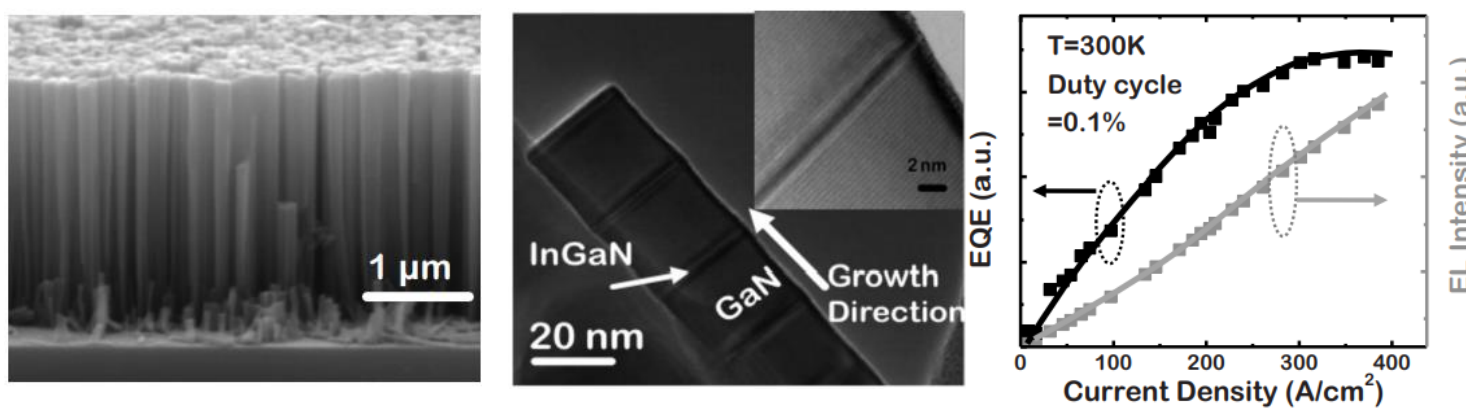

Fig. 4.5: (a) SEM image of high density GaN nanowires grown on Si substrate (b) High resolution TEM image of InGaN/GaN disk-in-a-wire heterostructure. (c) External quantum efficiency and EL intensity vs current injection of InGaN/GaN disk-in-a-wire LEDs [80]. 


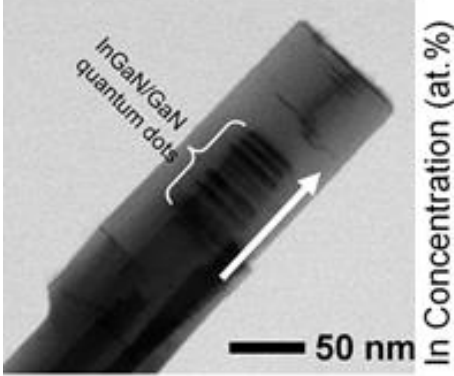

(a)

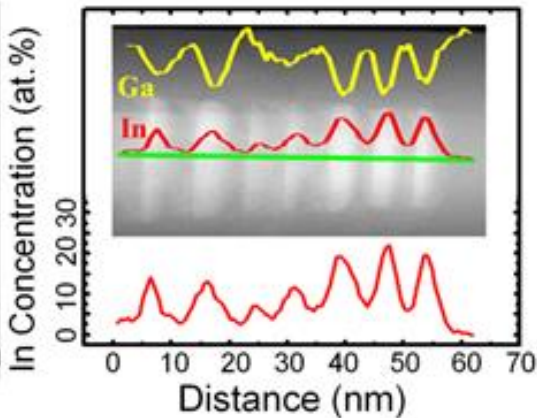

(b)

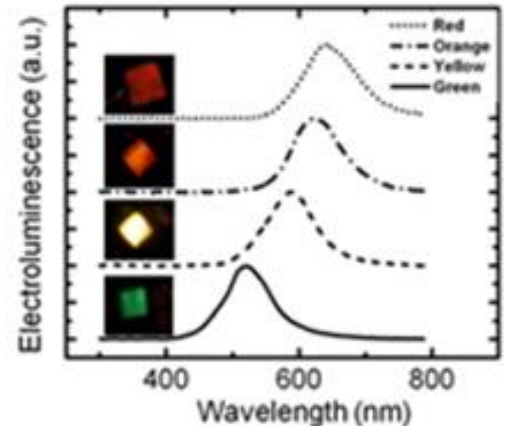

(c)

Fig. 4. 6: (a) STEM image of a single InGaN/GaN dot-in-a-wire showing multiple InGaN quantum dots in the middle of GaN wire. (b) EDX line scans show the variation of In along the InGaN dots. (c) EL spectra of different LEDs with different In composition in the dot active regions [36].

GaN dots are precisely positioned at the center of GaN nanowire due to strain-induced selforganization. This unique structure offers two distinct benefits comprising the greatly efficient carrier confinement in the active region and the self-surface passivation to reduce surface nonradiative recombination. Moreover, the emission color and color properties can be controlled by varying the compositions and/or the sizes of the dots. Figure 4.6(a) shows a bright field scanning transmission electron microscopy (STEM) image of a single InGaN/GaN dot-in-a-wire showing that multiple InGaN quantum dots are well positioned in the center of the wire. The dots are inserted in the center of the nanowires, thereby leading to highly efficient carrier confinement. Figure 4.6(b) shows the In concentration in each $\operatorname{In}_{x} \mathrm{Ga}_{1-x} \mathrm{~N}$ dot. The In composition $(x)$ in each dot is not uniform and varies from $\sim 10$ to 50 atomic percent. These nanowire LEDs exhibit strong PL intensities at $\sim 545 \mathrm{~nm}$ and very broad FWHM of $\sim 150 \mathrm{~nm}$, which was achieved by varying the In content in the quantum dots by adjusting growth temperatures and/or In/Ga flux ratios. Full-color emission was also developed based on this unique dot-in-a-wire nanostructure. Shown in Figure 4.6(c), the peak emission wavelengths are at $522 \mathrm{~nm}$ (green), $589 \mathrm{~nm}$ (yellow), $619 \mathrm{~nm}$ (orange), and $641 \mathrm{~nm}$ (red). Employing multiple color emission in a single nanowire, Nguyen et $a l$. has also demonstrated phosphor-free white
LEDs with well-defined white light characteristics.

\section{1) p-Type modulation doping for the enhanced hole transport}

Recent studies have shown that the poor hole transport severely limit the performance of GaN based LEDs. The holes injected to the GaN nanowire LEDs are mostly resided close to the $p$-GaN region because of large effective mass and low mobility. This nonuniform carrier distribution mainly in the device active region leads to enhance Auger recombination and increase electron overflow [101]. Nguyen et al. showed the improvement on hole transport and injection process using the $p$-doping in the device active region [36]. The improved hole transport results in significantly enhanced IQE. The $p$ doped dot-in-a-wire LEDs fabricated by Nguyen et al. shows high IQE under electrical injection at room temperature $\sim 56.8 \%$, presented in Figure 4.7. Compared to the undoped dot-in-a-wire LEDs, the IQE of $p$-doped nanowire LEDs was significantly enhanced. The $p$-doped dot-in-awire LEDs exhibit a maximum IQE of $\sim 50.2 \%$ under optical pumping, shown in the inset. The reported IQEs are drastically higher than any nanowire LEDs which is previously proposed. The higher IQEs are mainly attributed to the enhanced hole transport in the $p$-doped dot-ina-wire LEDs. The enhanced hole transport also 


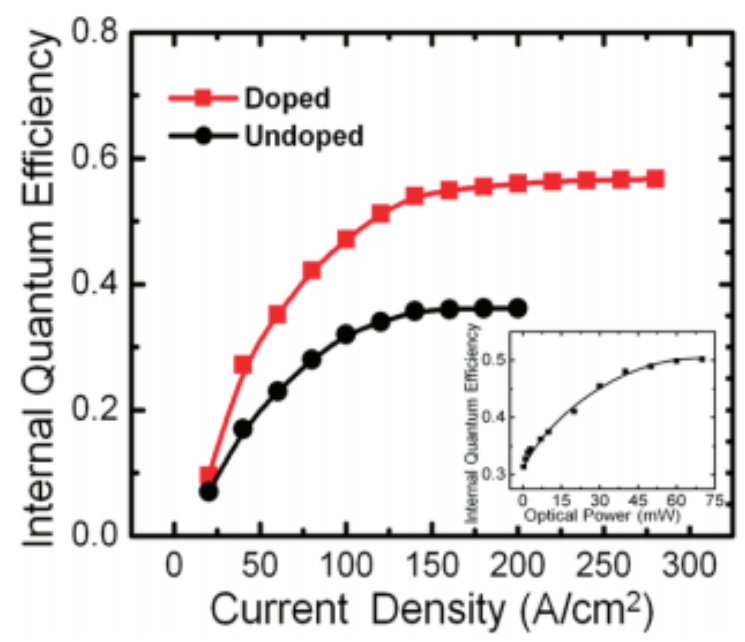

Fig. 4. 7: The IQE of the $p$-doped dot-in-a-wire LEDs at the room temperature is significantly enhanced compared to undoped one. The IQE of the $p$-doped dot-in-a-wire LED shows $\sim 50 \%$ under optical pumping are presented in the inset [36].

leads to electron overflow reduction, and less Auger recombination [36].

\section{2) Integrated AlGaN electron blocking layer in nanowire LEDs to reduce/eliminate electron overflow}

The performance of nanowire LEDs is more susceptible to electron leakage out of the device active region due to large densities of defects along the nanowire, compared to the conventional planar heterostructures. The resulting carrier loss and nonradiative carrier recombination severely reduce the quantum efficiency at higher injection currents. The electron overflow has been mitigated by utilizing the high bandgap AlGaN EBL inserted next to the $p$-GaN side of nanowire LEDs [51].

Nguyen et al. explained the role of electron overflow and how it limits the performance of nanowire LEDs [51]. They achieved truly white light emission incorporating InGaN dots with different In compositions. They demonstrated that the electron overflow can be significantly reduced by using large bandgap AlGaN electron blocking layer between the active region and the $p$-GaN section. They reported for the first time

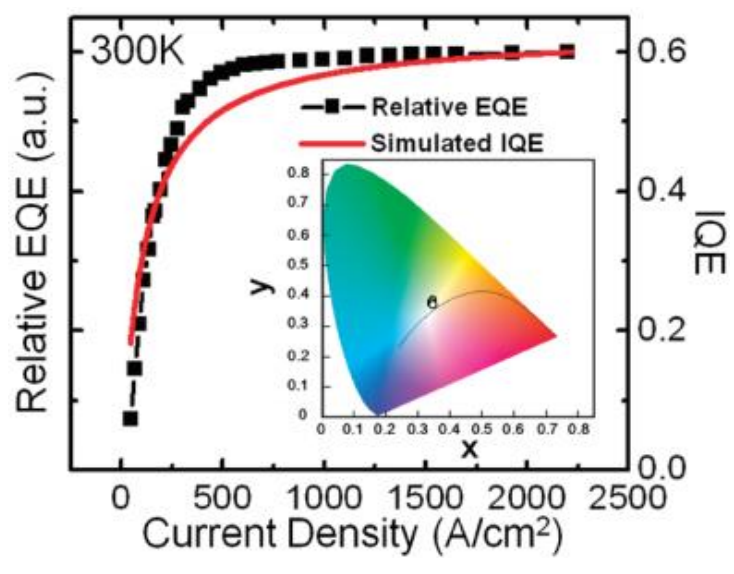

Fig. 4. 8: Relative external quantum efficiency of the In$\mathrm{GaN} / \mathrm{GaN}$ dot-in-a-wire LED with the AlGaN EBL shows no efficiency droop at room temperature [51].

the nanowire LEDs with virtually no efficiency droop up to $\sim 2200 \mathrm{~A} / \mathrm{cm}^{2}$ as shown in Figure 4.8 .

3) Core-shell nanowire LED structure for the reduced nonradiative surface recombination and enhanced carrier injection efficiency

Addition to the high bandgap AlGaN EBLs, core shell nanowires are alternative designs to reduce the electron overflow. The strong carrier confinement is the most important feature of coreshell nanowires which results in nanowire LEDs with very high emission efficiency. Nonradiative surface recombination has been among major responsible for the low quantum efficiency which is described in previous sections. Different surface passivation methods such as in situ grown large bandgap shell can be effectively used to improve the device performance $[83,84,86]$. Tchernycheva et al. demonstrated the nanowire InGaN/GaN core shell LEDs grown by MOVPE [102]. They proposed the core shell nanowire LED as shown in Figure 4.9 with the radial quantum well on the $m$-planes which showed light emission from green to blue with different injection currents. They obtained the EL with the light emission at $494 \mathrm{~nm}$ under low injection 

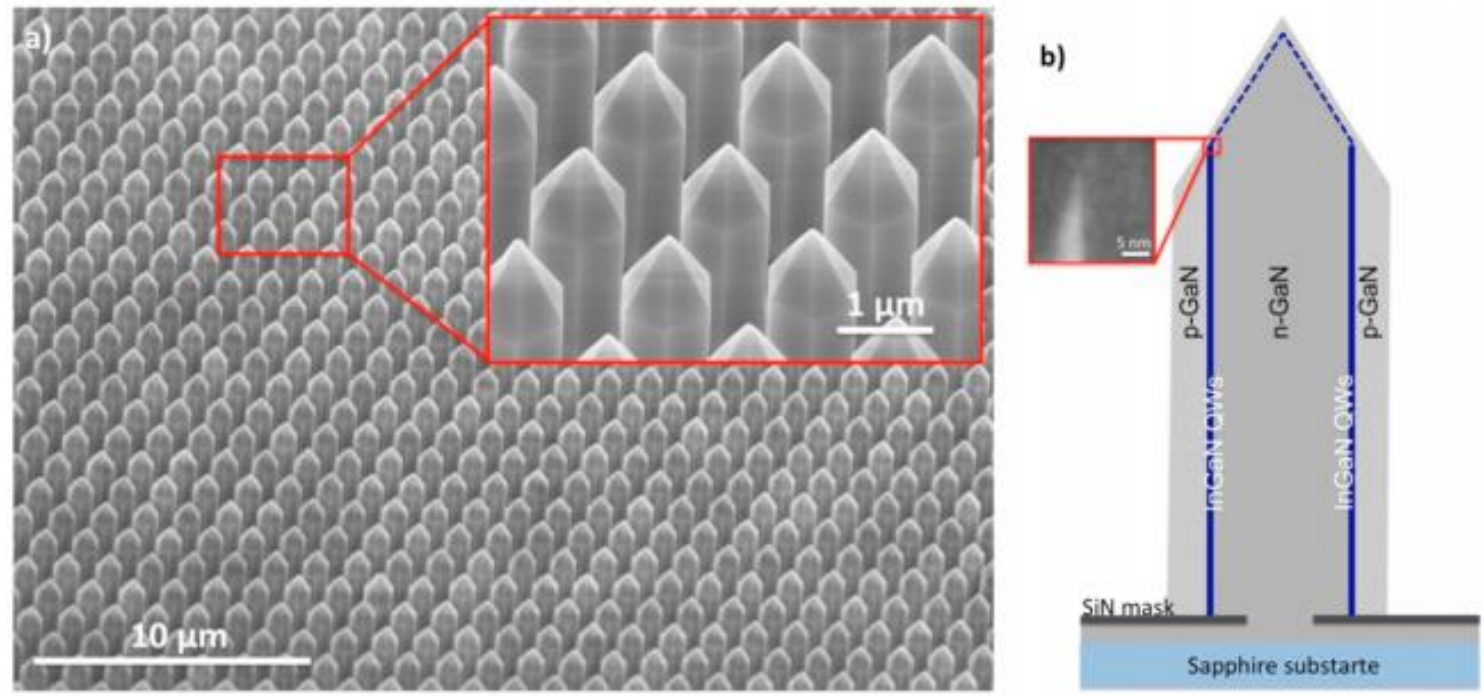

Fig. 4. 9: (a) SEM image of a core-shell nanowire LED with the radial quantum well on the $m$-planes. (b) Schematic structure of the core-shell nanowire [102].
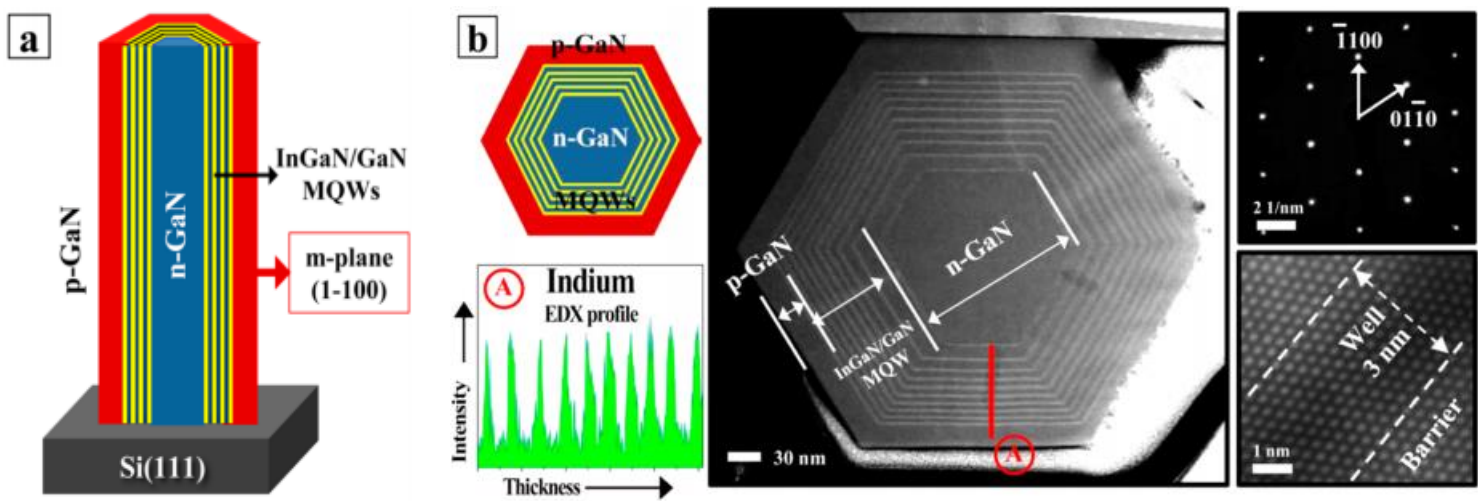

Fig. 4. 10: (a) The schematic diagram of In GaN/GaN MQW core-shell nanowire LED grown on Si(111) substrate. (b) High resolution TEM image of the InGaN/GaN core-shell nanowire [103].

current and the light emission at $415 \mathrm{~nm}$ under high injection current.

Ra et al. reported the core-shell nanowire LEDs in which the $n$-GaN core is surrounded by 10 pairs of $\mathrm{InGaN} / \mathrm{GaN}$ and $p$-GaN shell [103]. The schematic diagram and high resolution TEM image are shown in the Figure 4.10. They showed the improvement in the performance of core-shell nanowire LEDs because of reduced piezoelectric polarization fields. The core-shell nanowire LEDs proposed by Ra et al. exhibit a sharp onset voltage at $2.2 \mathrm{~V}$ with the high brightness light emissions. Nguyen et al. recorded that carrier injection efficiency of nanowire LEDs can be massively enhanced by employing an AlGaN shell covering the InGaN/GaN core to reduce the nonradiative recombination on the surface of nanowire LEDs [85]. The schematic structure of the $\mathrm{InGaN} / \mathrm{GaN} / \mathrm{AlGaN}$ dot-in-a-wire core-shell LED is shown in Figure 4.11(a). The structure comprising GaN:Si, ten vertically coupled $\mathrm{InGaN} / \mathrm{GaN}$ dots, a $\sim 10 \mathrm{~nm}$ $p$-doped AlGaN EBL, and GaN:Mg sections. A $\sim 80 \mathrm{~nm}$ AlGaN layer was grown finally grown to form a shell which covers the top as well as the lateral surfaces of the dot-in-a-wire structure. The large bandgap energy AlGaN shell can effectively reduce the nonradiative surface recom- 
(a)

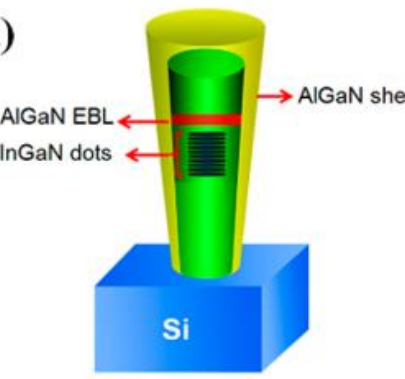

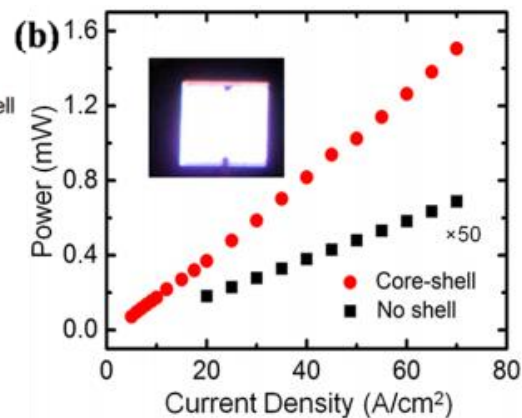

(c)

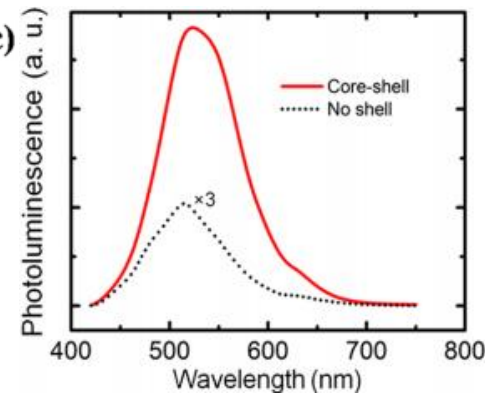

Fig. 4.11: (a) The schematic illustration of InGaN/GaN dot-in-a-wire core-shell LED heterostructures, (b) the output power of InGaN/GaN dot-in-a-wire LEDs with (red circle) and without (black square) the AlGaN shell under different injection currents, (c) The photoluminescence spectra of InGaN/GaN dotin-a-wire LEDs with (red solid) and without (black circle) the AlGaN shell [85].
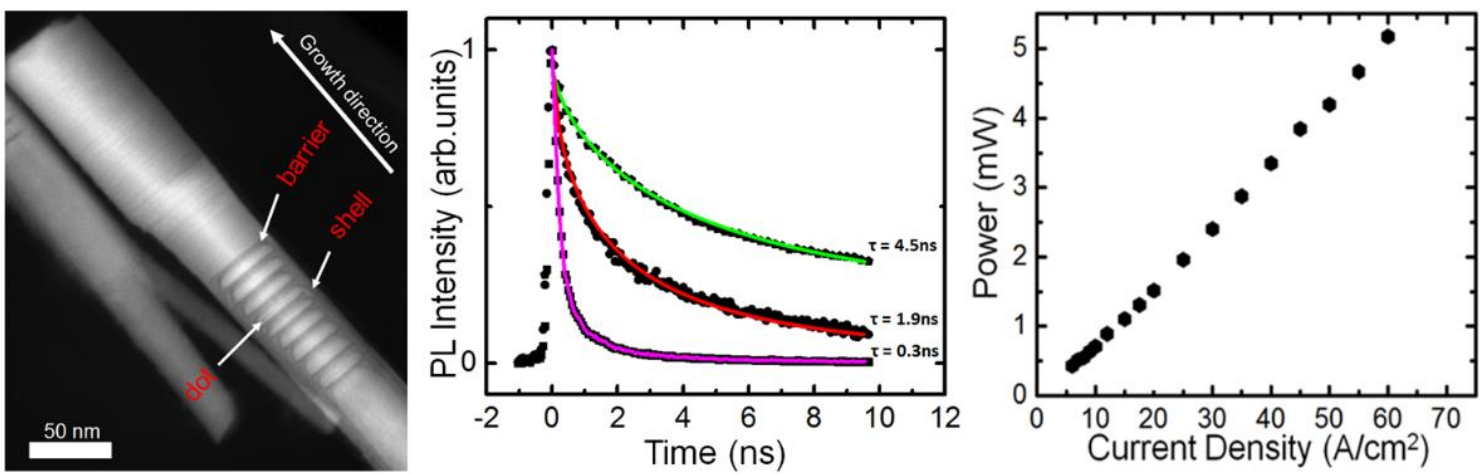

Fig. 4.12: (a) High resolution transmission electron microscope image of an In GaN/AlGaN nanowire LED structure. (b) Room temperature time-resolved photoluminescence emission of InGaN/AlGaN dot-in-a-wire core-shell (green and red curves) and InGaN/GaN nanowire LED heterostructures, showing the drastically increased carrier lifetimes in the core-shell LED samples. (c) Output power of the InGaN/AlGaN core-shell LEDs vs. different injection current densities [97].

bination, leading to increased carrier recombination in the active region. This enhances the output power and the measured output power for an injection current of $\sim 70 \mathrm{~A} / \mathrm{cm}^{2}$ is of $\sim 1.5 \mathrm{~mW}$, which is more than 50 times stronger than that of the InGaN/GaN nanowire LED without AlGaN shell and as shown in Figure 4.11(b). Stronger PL was observed for the corehell structure compared to the other and is provided in Figure 4.11(c). The greatly enhanced output power is attributed to the increased electrical injection efficiency, due to the drastically reduced nonradiative surface recombination resulted from the usage of efficient core-shell structure.

Nguyen et al. reported a unique InGaN/AlGaN core-shell nanowire LED with significantly reduced electron overflow, presented in the high resolution TEM image in Figure 4.12(a) [97]. They suggested that multiple AlGaN shells are spontaneously form during the growth of the AlGaN barriers in the active region. The AlGaN shell formed on the sidewall leads to significantly reduce the nonradiative surface recombination. The carrier injection efficiency is also remarkably increased due to the strong carrier confinement offered by the large bandgap AlGaN shell. The derived carrier lifetime is significantly enhanced from $0.3 \mathrm{~ns}$ to $4.5 \mathrm{~ns}$, due to the reduced surface recombination, shown in Figure 4.12(b). They demonstrated that the AlGaN barrier layers between quantum dots act as distributed EBLs effectively reduce the electron overflow. They obtained the core-shell LEDs which emit strong white light emission with an 

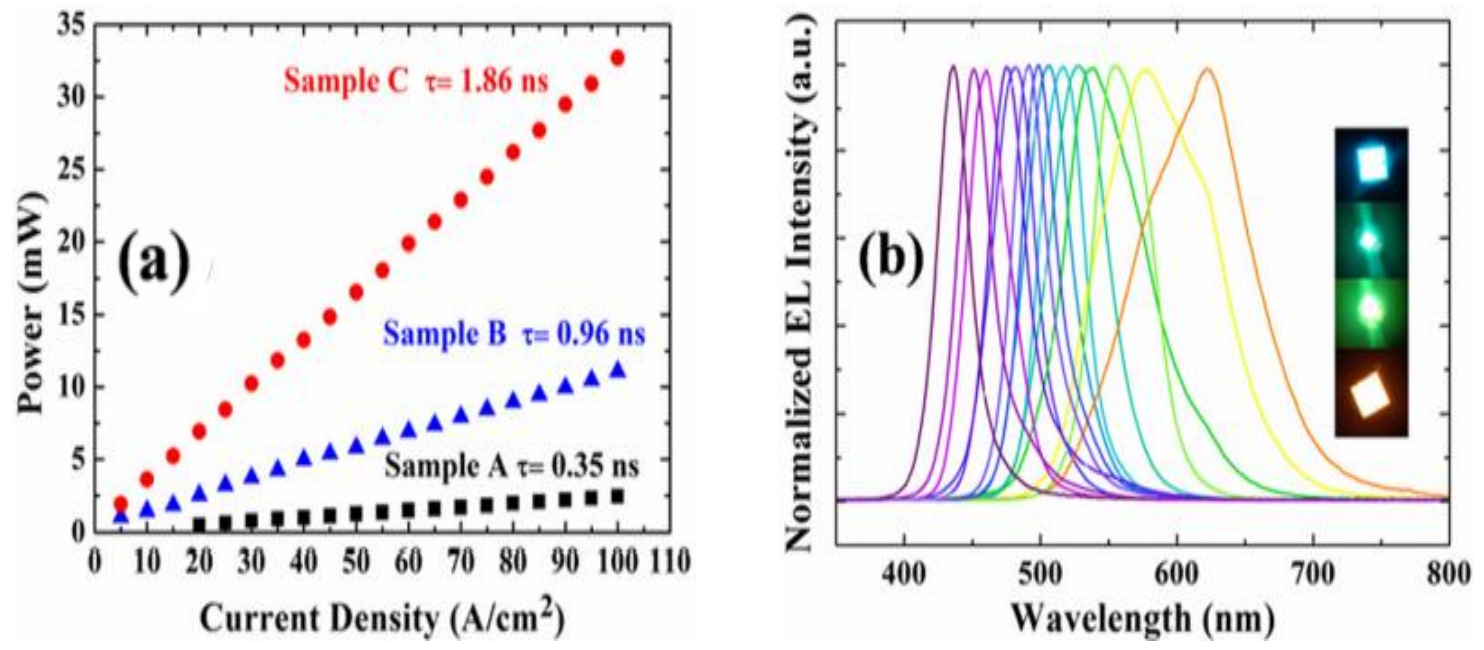

Fig. 4. 13: (a) Measured output power vs. carrier lifetime of AlInGaN core-shell nanowire LEDs grown at different growth conditions. (b) Normalized EL spectra of different AlInGaN core-shell nanowire LEDs with their optical images are illustrated in the inset [104].

output power of more than $5 \mathrm{~mW}$, shown in Figure $4.12(\mathrm{c})$.

Wang et al. proposed AlInGaN core-shell nanowire LED structures with a spontaneously formed Al-rich shell [104]. The carrier lifetime was significantly enhanced (up to $\sim 2 \mathrm{~ns}$ ) for nanowire structures with a well-defined AlGaN shell. The output power (measured at $90 \mathrm{~A} / \mathrm{cm}^{2}$ ) increased dramatically for nanowire devices with a longer carrier lifetime as shown in Figure 4.13(a), due to the significantly reduced nonradiative surface recombination and enhanced carrier injection efficiency. Moreover, the emission wavelengths can be tuned from $\sim 430 \mathrm{~nm}$ to $630 \mathrm{~nm}$, shown Figure 4.13(b).

\subsection{Polarization-induced $p$ - $n$ junction nanowire LEDs}

Simon et al. reported that the $p$-doping in IIInitrides can be greatly enhanced by ionizing acceptor dopants using the built-in polarization field in bulk uniaxial semiconductor [105]. This concept enables a new type of nanowire LEDs in which the $p$ - $n$ junction is not formed by conventional impurity doping approach, but rather by polarization-induced $p$ - $n$ junction [106]. The structure of such nanowire LED, reported by Carnevale et al. [106], includes an active region of single GaN quantum disk, AlN cladding layers and AlGaN grading layers on both sides of the well, as shown in Figure 4.14 (a), an $\mathrm{Al}_{x} \mathrm{Ga}_{1-x} \mathrm{~N}$ layer to form the $n$-conducting segment in which $\mathrm{x}$ varies linearly from 0 to 1 along the [0001] direction, and an $\mathrm{Al}_{x} \mathrm{Ga}_{1-x} \mathrm{~N}$ grading layer with $\mathrm{x}$ reduces from 1 to 0 for the formation of $p$-type conducting region, resulted in the $p$ - $n$ junction without using impurity doping. Figure 4.14 (b) shows the modeled energy band diagram of the nanowire LED along its length. The currentvoltage characteristics and the EL spectra of the polarization-induced nanowire LEDs are shown in Figure 4.14 (c). The emission energy shows strong blue-shift from $2.4 \mathrm{eV}$ to $3.29 \mathrm{eV}$ with the increased injection current. At high bias, the polarization charge can be screened, the redshift of EL cause by quantum confined Stark effect can be reduce, leading to strong blue shift. Such new nanowire LEDs were further investigated and reported using mixed polarity concept in polarization-induced nanowire LEDs [107].

\subsection{Tunnel junction-induced nanowire LEDs}

III-nitride material-based LEDs have resistive $p$ type layers which leads to have poor ohmic contact. In addition, due to high activation ener- 

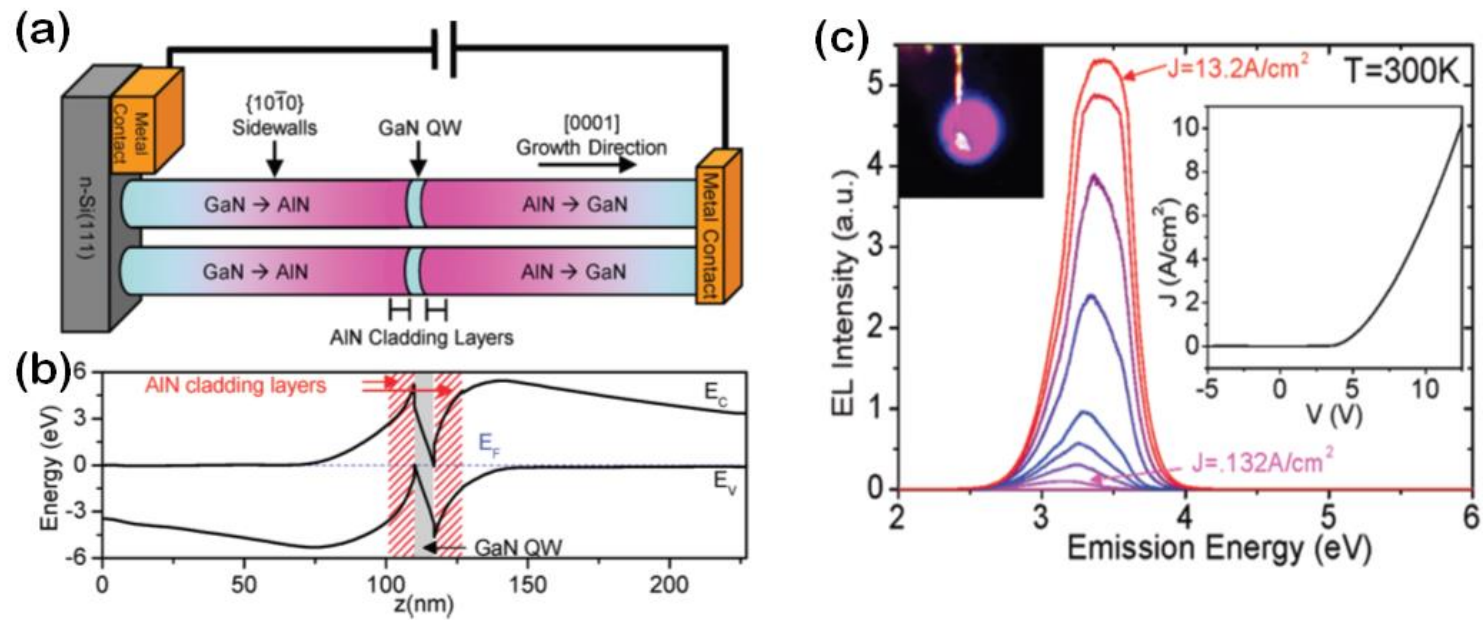

Fig. 4.14: (a) Schematic of a polarization-induced $p$ - $n$ junction LED. (b) Modeled energy band diagram of the LED along the length of the nanowire. (c) Electroluminescence spectra of the nanowire LED and current-voltage characteristic of such nanowire LED [106].

gies of $\mathrm{Mg}$ dopants, it is difficult make heavily doped $p$-GaN layer. These create serious hole transportation problem in LEDs. This problem can be reduced with the incorporation of tunnel junction (TJ) in the LED. When a TJ is integrated with single active region LED, LED can be forward biased while the TJ is operating in reverse biased condition. In this case, TJ works as carrier conversion center which supplies holes to the active region, due to this $p$-GaN can be replaced by $n$-GaN. Sharif et al. reported that the monolithically integrated metal/semiconductor TJ based nanowire LED where $n^{++} \mathrm{GaN} / \mathrm{Al} / p^{++} \mathrm{GaN}$ considered as TJ, schematic diagram is provided in Figure 4.15 (a) [108]. Due to very high $M g$-doping, defects are present at the $\mathrm{Al} / p++$-GaN interface, which can dramatically improve the carrier transport from $p^{++} \mathrm{GaN}$ to $\mathrm{Al}$ in a similar manner to conventional trap-assisted tunneling. As a result, effective tunneling barrier width reduces which enhances the carrier transportation. They reported that the Al-TJ based nanowire LEDs have better performance compared to conventional TJ $\left(n^{++} \mathrm{GaN} / p^{++} \mathrm{GaN}\right)$ based nanowire LEDs, corresponding schematic energy band diagrams are presented in Figure 4.15 (b). Shown in Figure 4.15 (c), Al-TJ based nanowire LED exhibited better I-V characteristics with a sharp turn-on voltage of $\sim 2.9 \mathrm{~V}$ at strong green light emission compared to conventional TJ and con- ventional dot-in-a-wire nanowire LEDs. Moreover, Al-TJ based nanowire LED showed strong light intensity compared to other, shown in the Figure 4.15 (d).

Sharif et al. reported the first alternative current $(\mathrm{AC})$ operated nanowire LEDs on $\mathrm{Si}$ substrate with the integration of tunnel junction, which can operate with positive and negative polarities of applied voltage [109]. The demonstrated AC LED consists of $p$-GaN up (conventional dot-in-a-wire) and $p$-GaN down (tunnel junction-based dot-in-a-wire) nanowire LEDs are grown on same Si chip using selective area growth technique, schematic diagram is shown in the Figure 4.16 (a). The electrical measurements were performed at a peak to peak voltage of $10 \mathrm{~V}$ with $20-60 \mathrm{~Hz}$ frequency. During the positive polarity of the applied voltage, $p$-GaN up LEDs are emitting light and $p$-GaN down LEDs are emitting light under the negative polarity applied voltage condition, it can be seen from Figure 4.16 (b). This technique avoids the use of AC to DC converter in the LED applications. 

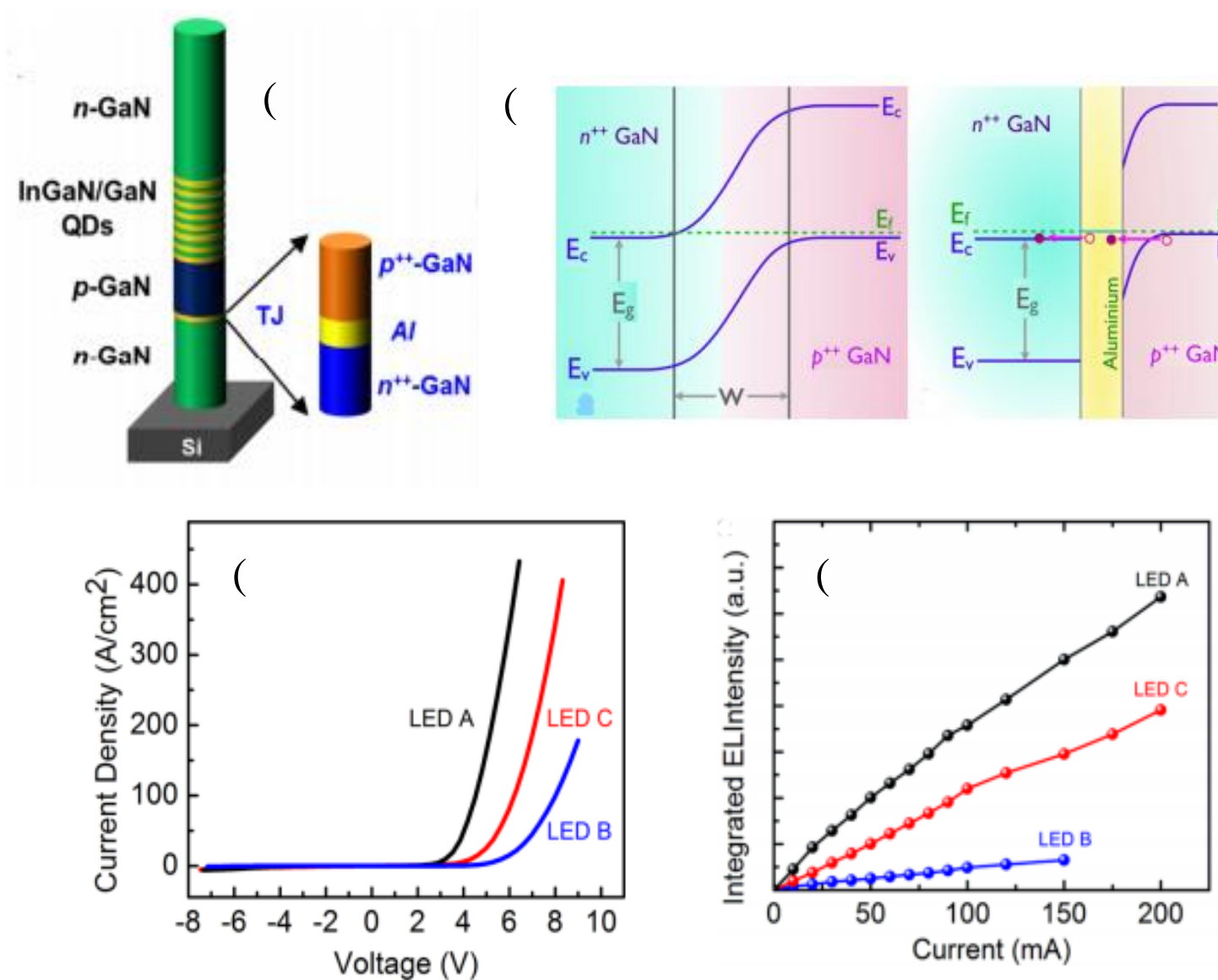

Fig. 4. 15: (a) Schematic diagram of Al tunnel junction-based nanowire LED. (b) Modeled energy band diagram of Al tunnel junction, conventional tunnel junction-based nanowire LEDs. (c) I-V characteristics of $\mathrm{Al}$ tunnel junction (LEDA), conventional tunnel junction (LEDB) and conventional dot-in-a-wire (LEDC) nanowire LEDs. (d) L-I characteristics of Al tunnel junction (LEDA), conventional tunnel junction (LEDB) and conventional dot-in-a-wire (LEDC) nanowire LEDs [108].

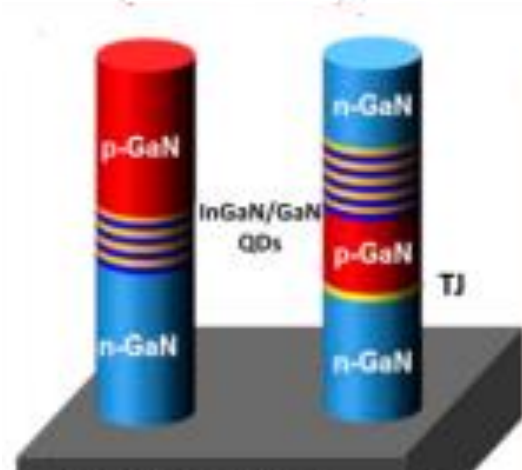

Si (111) substrate

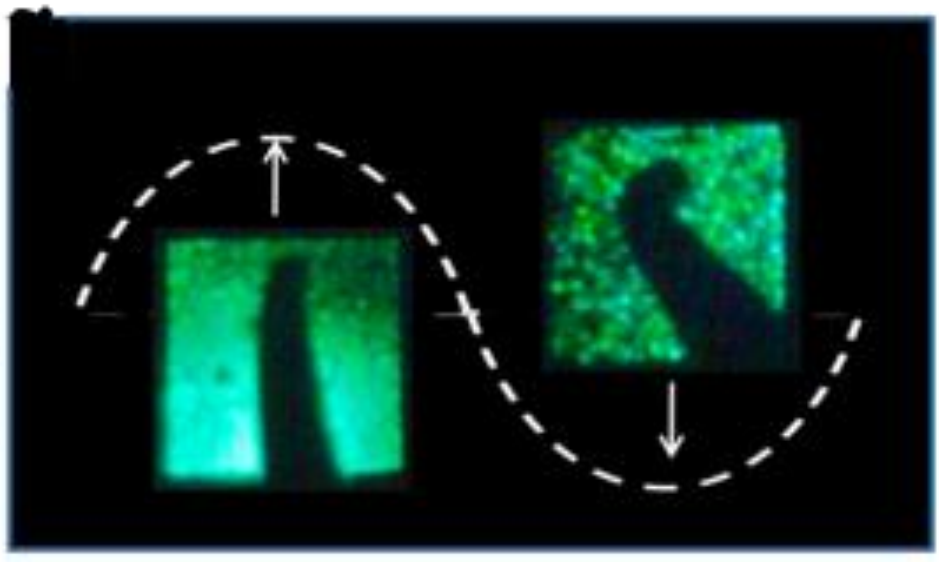

Fig. 4. 16: (a) Schematic diagram of AC nanowire LED on Si substrate. (b) Optical image of AC nanowire LED emitting green light under AC biasing conditions [109]. 

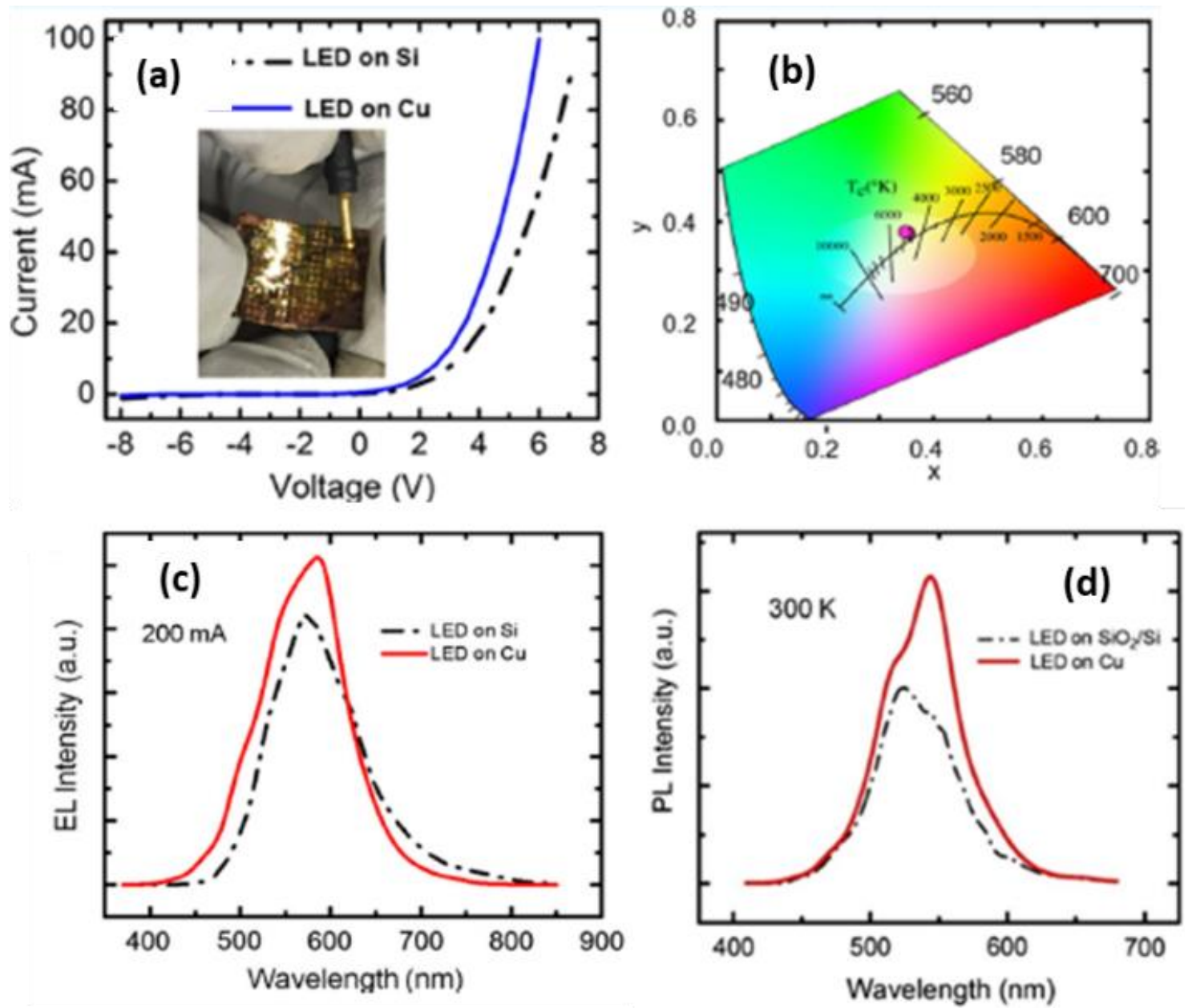

Fig. 4.17: (a) Current-voltage characteristics of the LEDs on the Si and on Cu substrates, (b) CIE diagram of phosphor-free white LEDs on the $\mathrm{Cu}$ substrate [113], (c) EL spectra of LED structures on $\mathrm{SiO}_{2} / \mathrm{Si}$ (dash-dot curve) and on $\mathrm{Cu}$ (solid curve) substrates, and (d) PL spectra of LEDs on $\mathrm{Cu}$ and $\mathrm{SiO}_{2} / \mathrm{Si}$ substrates [112].

\subsection{III-Nitride nanowire LEDs on metal substrates}

The development of high-power III-nitride nanowire LEDs is limited partly in term of native substrates for the epitaxial growth and using metal substrates is a viable solution. Although III-nitride nanowire LED structure grown on foreign substrates like sapphire, silicon, and silicon carbide has demonstrated considerable advantages over its planar counterpart in term of reduced dislocation and strain caused by the lattice mismatch. However, when it comes to highpower LED applications, poor heat dissipation in disrupted nanowire arrangements on an alien substrate and its optical absorption need to be overcome. In this context, the metal substrates such as copper $(\mathrm{Cu})$ or Molybdenum (Mo) are very investigated. Using metal substrates will provide the enhanced heat dissipation, high light reflectability, avoid optical absorption from the conventional substrates.

The III-nitride planar LEDs on $\mathrm{Cu}$ substrates with better performance than that on sapphire substrates was reported in 2004 [110, 111]. Similarly, Nguyen's group reported on highperformance III-nitride nanowire LEDs on copper $(\mathrm{Cu})$ substrates via the substrate-transfer process using combining dry- and wet-etching 

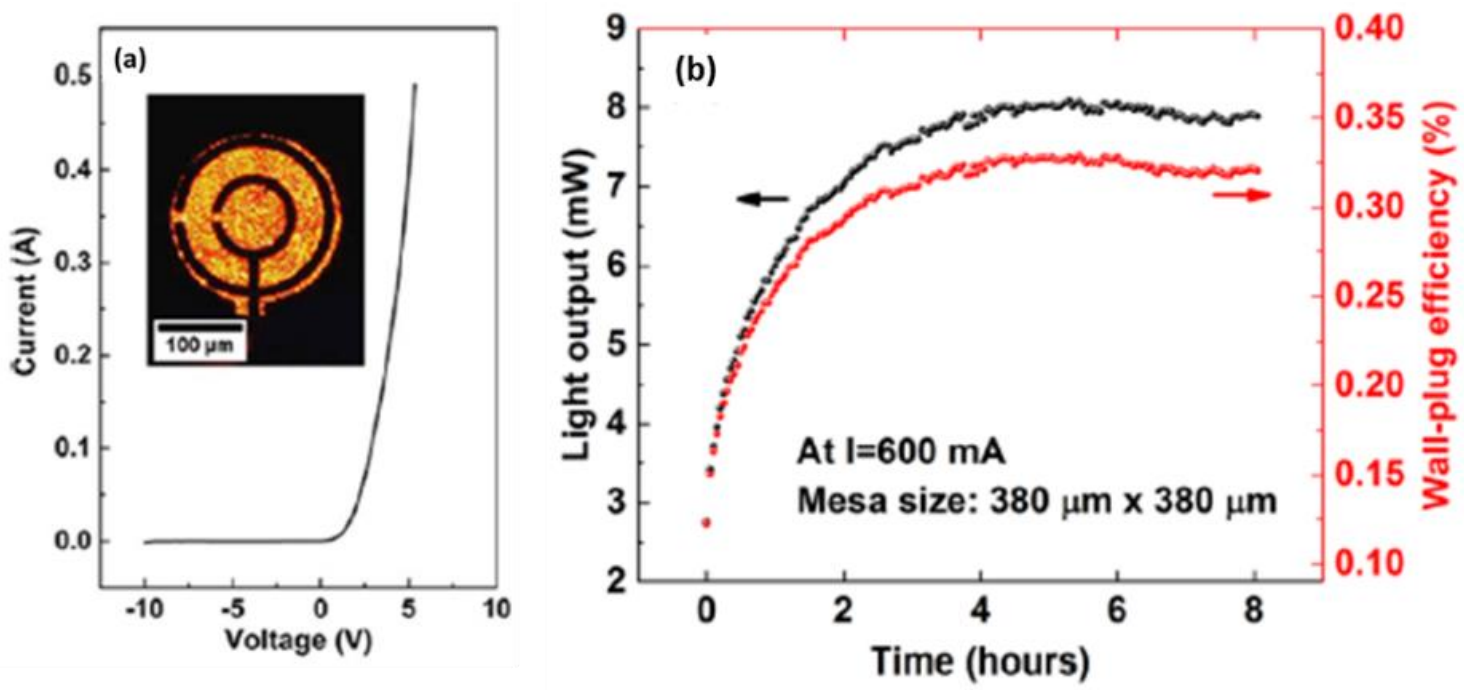

Fig. 4. 18: (a) Measured I-V characteristics of the $200 \mu \mathrm{m}$ diameter LED with the optical microscope image of the uniformly illuminated LED with a mesa diameter of $200 \mu \mathrm{m}$ in the inset [114]; (b) light output power and wall-plug efficiency of LEDs on Mo versus characterization time. A constant current of $600 \mathrm{~mA}$ was applied to the LEDs with size of $380 \mu \mathrm{m} \times 380 \mu \mathrm{m}[115]$.

processes [112, 113] and shown in Figure 4.17. The nanowire LEDs on $\mathrm{Cu}$ show more efficient thermal management and enhanced LEE than the nanowire LEDs on conventional silicon substrate. The advantages can be attributed to metal reflectors and high thermal conduction of $\mathrm{Cu}$. The resulting PL, PL intensities, and current-voltage characteristics of nanowire LEDs on $\mathrm{Cu}$ substrate are higher than that of $\mathrm{Si}$ substrates. These better performances were solidified by the simulation. The LEE of the nanowire LED on $\mathrm{Cu}$ is nine times higher than that of the LED on $\mathrm{Si}$ at the same nanowire radius of $60 \mathrm{~nm}$ and spacing of $130 \mathrm{~nm}$. Moreover, the LEDs on $\mathrm{Cu}$ substrates present highly stable white-light emission with high color-rendering index of $\sim 95$, promising in applications in general lighting, flexible displays, and wearable applications.

Titanium ( $\mathrm{Ti}$ ) and Mo coated conventional substrates used for the growth of GaN nanowire LEDs have been reported recently by Wolz et al. and Sarwar in order to improve heat dissipation and current injection for high power nanowire LEDs. Zhao et al. demonstrated a direct growth of InGaN/GaN quantum-disk nanowire LEDs on commercial Mo substrates [114]. The turn-on voltage of the resulting LEDs is relatively low at $\sim 2 \mathrm{~V}$ and the efficiency droop is unnoticeable up to injection current of $500 \mathrm{~mA}\left(1.6 \mathrm{kA} / \mathrm{cm}^{2}\right)$ at $\sim 5 \mathrm{~V}$ [115], shown in Figure 4.18. As a result, high-power nanowire LEDs on metal substrates could be beneficial for multiple cross-disciplinary applications thanks to the chemical stability of nitride materials, high surface to volume ratio of nanowires, flexibility, high-temperature operation.

\section{Visible light emitters}

LEDs with color tunability are essential for various ranges of applications such as smart-lighting [116], and displays [117]. The white LEDs utilizing phosphor converters have limited performance because of their low CRI and Stokes fluorescence loss [118]. Full-color nanowire LEDs operating in the visible wavelength region and phosphor-free white-color nanowire LEDs are presented below. 


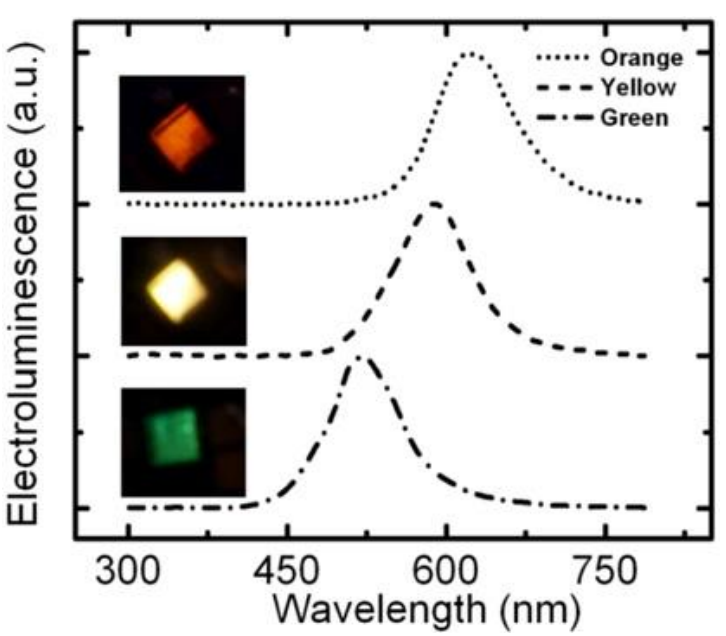

Fig. 5.1: The electroluminescence spectra of GaN nanowire LEDs at room temperature which shows green, yellow and orange light emissions and their optical microscopy images (insets) [50].

\subsection{Color tunable nanowire LEDs with emission wavelengths in the red, green, and blue regions}

Monolithically integrated RGB LEDs enable the spectral tuning to achieve the green, yellow and red which can lead to full-color LEDs with low power consumption, extremely small size, and high color rendition. The lattice mismatched between the substrates and the high-In InGaN quantum wells results in very low efficiency GaN-based LEDs in the green and longer wavelength ranges. However, nanowire LED structures offer ability to achieve high-efficiency emission in the entire visible spectral range. Nguyen et al. demonstrated such GaN nanowire LEDs with high efficiency green, yellow and orange LEDs as shown in Figure 5.1. Varying the growth temperature, the In/Ga flux ratio as well as the growth duration are among the factors to control the emission wavelengths. Moreover, they demonstrated the phosphor-free InGaN/GaN dot-in-a-wire white LEDs mixing different colors emitted from large inhomogeneous broadening of the InGaN/GaN quantum dots. They showed that the IQE of GaN nanowires LEDs could reach up to $\sim 22 \%$ at room temperature [50].

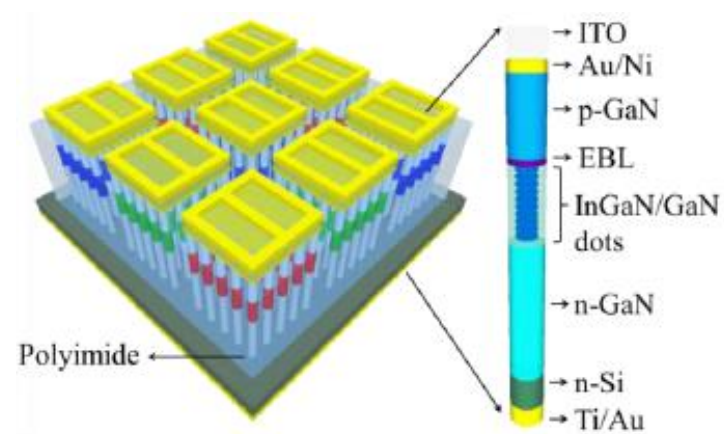

Fig. 5. 2: Schematic illustration of the tunable full color GaN LEDs utilizing integration of blue, green/yellow, and orange/red light emissions on the same chip [119].

Wang et al. demonstrated the tunable full color GaN-LEDs utilizing integration of blue, green/yellow, and orange/red light emissions as shown in Figure 5.2 [119]. In their experiment, the GaN nanowire LEDs were grown directly on Si substrate by three step selective area MBE process using $\mathrm{SiO}_{2}$ mask. The EL spectra of nanowire LED subpixels grown at different steps are shown in Figure 5.3 (a). The emission properties of each nanowire LED could be controlled by adjusting the dimension and/or compositions of the dots. Such LED arrays on the same chip exhibit color-tunable characteristic in a wide range $\mathrm{CCT}$ which is from $3800 \mathrm{~K}$ to $6500 \mathrm{~K}$ by applying independent injection currents to each LED subpixel. Moreover, these integrated tunable full-color LED arrays can also exhibit very high color rendering index $(>90)$, shown in Figure 5.3 (b). These small size RGB nanowire LED arrays offer highly promising applications for future smart lighting, and displays.

Recent studies have presented that the emission color of nitride-based nanowire LEDs can be varied by controlling the composition of the InGaN active region through the changing nanowire diameters as shown in Figure 5.4 [120, 121]. Sekiguchi et al. demonstrated GaN nanowire LEDs utilizing Ti-mask selective area MBE growth [120]. They reported the GaN nanowire LEDs which exhibit different light emission ranges from $480 \mathrm{~nm}$ to 632 $\mathrm{nm}$ with different diameters from $143 \mathrm{~nm}$ to 270 $\mathrm{nm}$. The peak emission wavelengths changed from blue $(480 \mathrm{~nm})$ to red $(632 \mathrm{~nm})$ with in- 


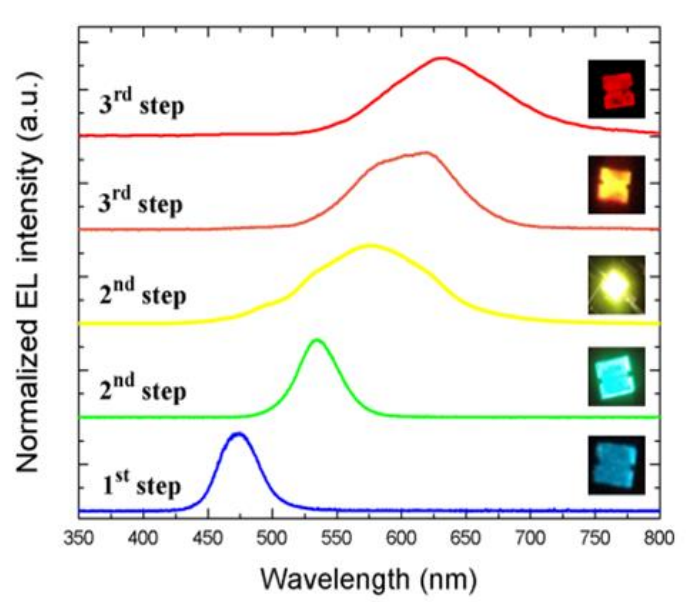

(a)

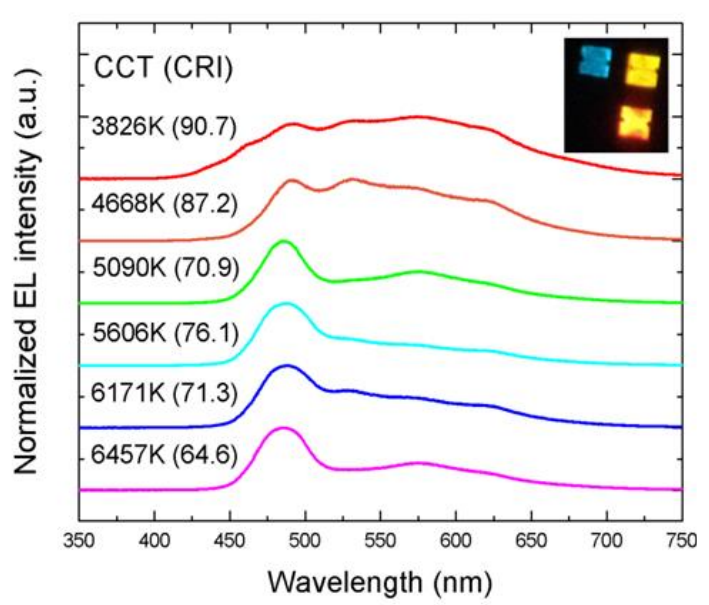

(b)

Fig. 5. 3: (a) Normalized EL spectra of nanowire LED subpixels monolithically integrated on Si substrate under the injected current of $20 \mathrm{~mA}$. The inset shows optical images of multi-color emissions from different nanowire LED subpixels monolithically integrated on Si substrate. (b) The output spectra and their corresponding $\mathrm{CCT} / \mathrm{CRI}$ of a representative triple-color LED pixel consisting of three $300 \times 300 \mu \mathrm{m}^{2}$ devices [119].

creasing nanowire diameter and can be seen from Figure 5.4.

Albert $e t$ al. showed nanowire LEDs with different emission wavelengths by varying the In$/ \mathrm{Ga}$, III/V ratios, and the growth temperature [122]. They further reported white light emission achieved by single nanowire mixing the redgreen-blue colors from different InGaN portions of the nanowire [122]. Recently, Wang et al. developed the AlInGaN quaternary core shell nanowire LEDs with tunable emission wavelengths from $\sim 430 \mathrm{~nm}$ to $\sim 630 \mathrm{~nm}$. They can significantly suppress the nonradiative surface recombination utilizing an Al-rich shell which enhance the output power $\sim 30 \mathrm{~mW}$ [104].

Recent studies have further shown that amorphous $\mathrm{SiO}_{x}$ can be used as substrate for superior quality nanowire LED heterostructures $[112,123,124]$, which pave the way for realizing high efficiency, flexible, and multi-color nanowire LEDs. Nguyen et al. demonstrated the direct transfer of $\mathrm{InGaN} / \mathrm{GaN}$ nanowire LEDs from $\mathrm{SiO}_{x} / \mathrm{Si}$ substrate to copper substrate [112] which exhibited improved currentvoltage characteristics and higher emission intensity compared to nanowire LEDs on Si. The enhanced performance of such devices is due to the reduced photon absorption by the underlying substrate and the strong heat dissipation.

\subsection{Phosphor-free nanowire white LEDs}

Even though significant progresses have been made for the conventional III-nitride planar quantum well white LEDs, these devices still suffers from several major performance limitations including severe efficiency droop at high injection current and significant reduced quantum efficiency in the green and even longer emission wavelength region, both of which are probably associated with strong polarization fields [82, 125], Auger recombination [3], inhomogeneous carrier distribution [67], defects/dislocation [126], and/or electron leakage [75]. The current white LED lighting utilize phosphors to down-conversion of blue light of an InGaN quantum well LED into green and red light. This significantly limits the device efficiency, increases the manufacturing complexity, and further compromises the device stability and light quality [127-130]. However, due to the effective strain relaxation, III-nitride nanowire LED structures can be utilized to produce high performance 

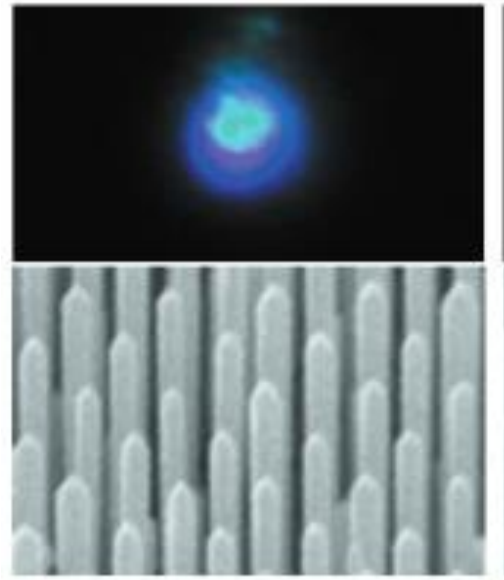

(a) $\mathrm{D}=143 \mathrm{~nm}$
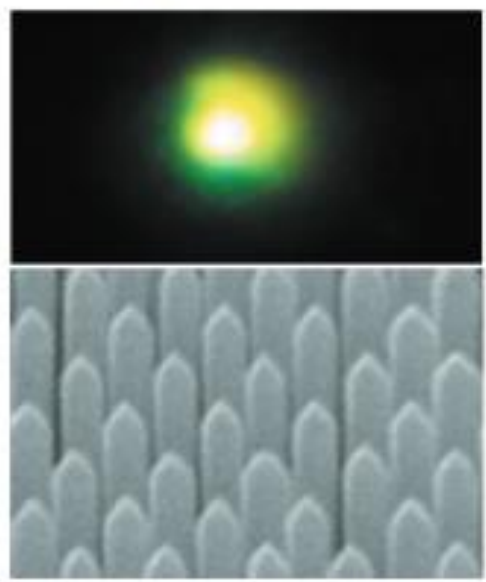

(d) $\mathrm{D}=196 \mathrm{~nm}$
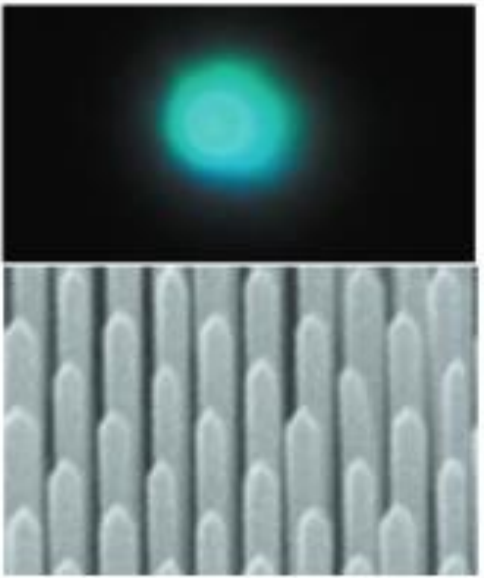

(b) $\mathrm{D}=159 \mathrm{~nm}$
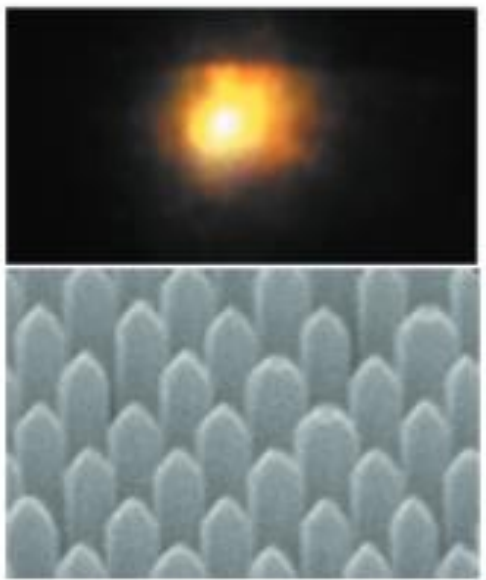

(e) $\mathrm{D}=237 \mathrm{~nm}$
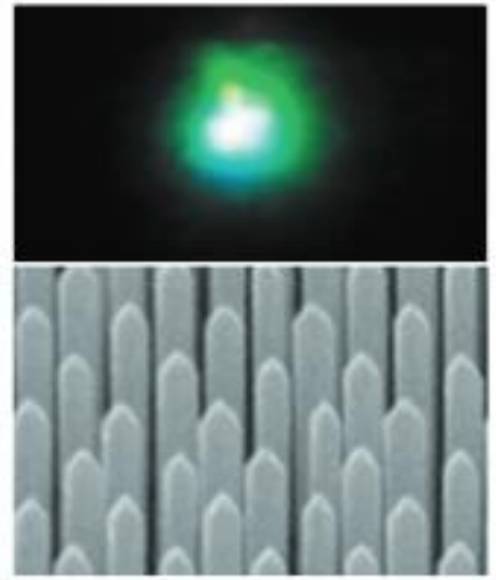

(c) $\mathrm{D}=175 \mathrm{~nm}$
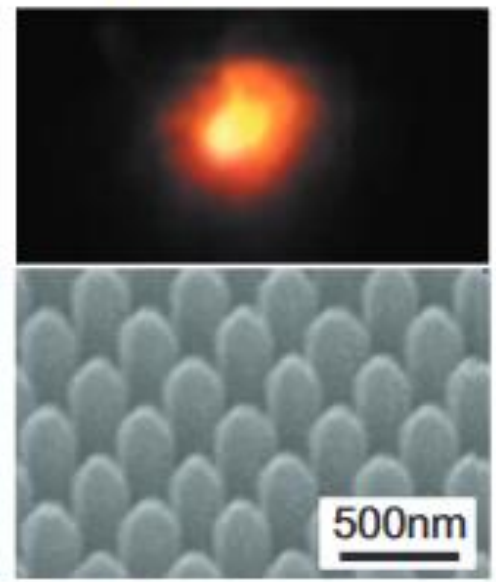

(f) $\mathrm{D}=270 \mathrm{~nm}$

Fig. 5.4: SEM images and corresponding PL optical images of InGaN/GaN nanowires with different diameters showing the different emission colors. The diameters are (a) $143 \mathrm{~nm}$, (b) $159 \mathrm{~nm}$, (c) $175 \mathrm{~nm}$, (d) 196 $\mathrm{nm}$, (e) $237 \mathrm{~nm}$, and (f) $270 \mathrm{~nm}[120]$.

phosphor-free white LEDs. This approach enables solid state lamps with the highest efficiency, longest lifetime, and best light quality, compared to any existing phosphor-based lighting technology. Moreover, significant progress has been made in nanowire LEDs and their performance has been improved by engineering the active regions with several nanostructure designs consisting of well/disk-in-a-wire [39, 120], core/shell [131-134], ternary nanowire [99, 135], and dot-in-a-wire nanoscale heterostructures [136] which were described in previous sections. These devices have already shown rel- atively high IQE, tunable emission across the entire visible spectral range, and droop-free operation [36, 99, 137]. Recently, InGaN nanowire LEDs has been demonstrated which show efficient light emission across nearly the entire visible spectral range [99, 138, 139].

Lin et al. proposed phosphor-free white LEDs using In GaN/GaN nanowire heterostructures by mixing multiple colors emitted from a single nanowire [139]. They reported that changing the growth temperature and In/Ga beam fluxes can tune the color emission. Figure 5.5(a) presents the PL spectra of InGaN nanodisk with differ- 
a

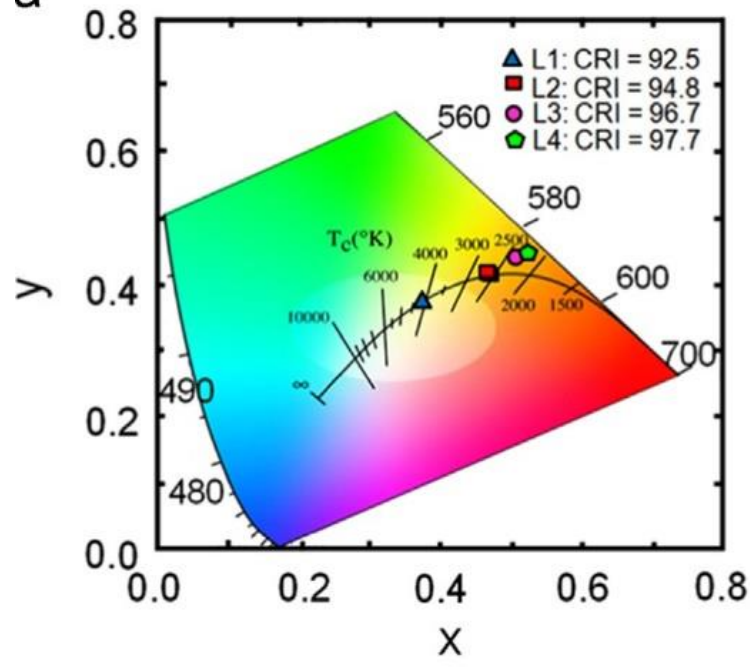

b

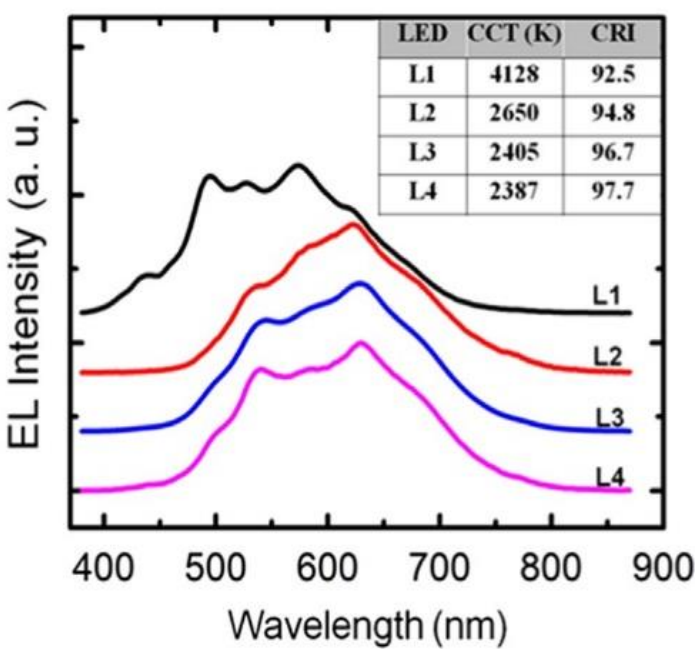

Fig. 5. 5: (a) Tuning the color emission of InGaN nanowire LEDs in the entire visible ranges with changing the growth condition, (b) The PL spectra of different LEDs showed that mixing light can produce the natural white emission with different CCT and CRI values [139].
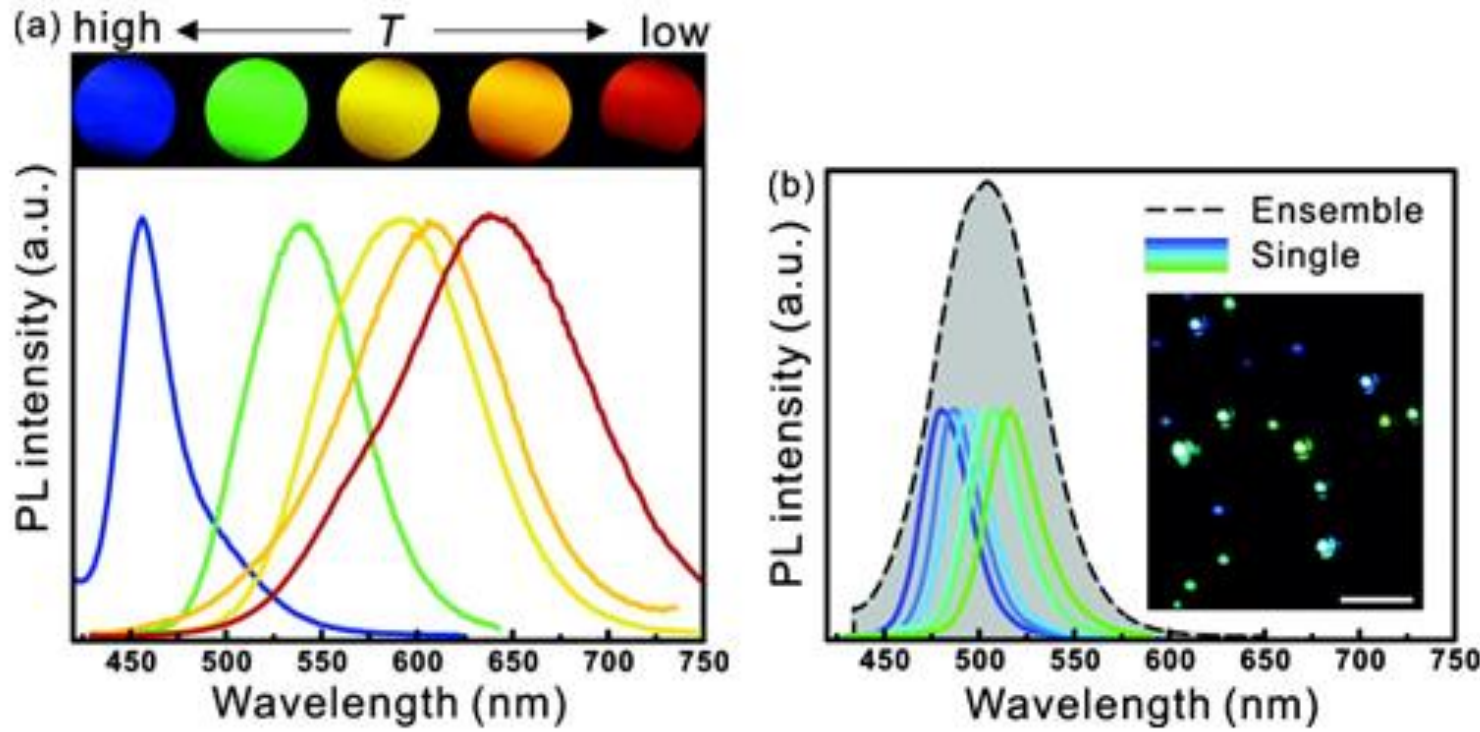

Fig. 5.6: (a) Photoluminescence spectra of InGaN nanowire LEDs grown at different conditions. (b) Photoluminescence spectra of different LEDs with peak emission wavelengths vary from blue to green, and the mixing light exhibits natural white emission centered at $504 \mathrm{~nm}[139]$.

ent growth temperature. In Figure 5.5(b), the InGaN nanodisk LED emissions cover the entire visible light range producing natural white light.

Guo et al. reported large area white LEDs using InGaN/GaN nanowire double heterostructures without the incorporation of any phosphor-converter [99]. Recently, Nguyen et al. demonstrated phosphor-free quantum dot nanowire LEDs in which white light emission can be achieved with various sizes and compositions of InGaN quantum dots in GaN nanowires [36, 40, 50]. They have further demonstrated core-shell nanowire white LEDs with an in-situ 
grown AlGaN shell which significantly enhanced output power by enhancing the carrier injection efficiency and suppressing nonradiative surface recombination [85]. The correlated color temperature (CCT) can be changed from $2000 \mathrm{~K}$ to $45000 \mathrm{~K}$ as shown in Figure 5.6(a). The proposed LED showed excellent color rendering index (CRI) with the highest quality warm and cool white generation. Figure 5.6(b) presents the emission spectra of phosphor-free whitecolor LEDs. They have also recently reported a unique self-organized InGaN/AlGaN dot-in-awire core-shell white LED heterostructure which is the most efficient axial nanowire core-shell LEDs [34]. Strong and stable white-light emission was recorded for those core-shell nanowire LEDs with a high CRI of $\sim 95$, with the $x$ and $y$ values in the ranges of $\sim 0.35-0.36$ and $0.37-0.38$, respectively. Such unique core-shell nanowire heterostructures are promising candidates for a broad range of photonic devices, including lasers, solar cells, and photodetectors.

\section{Conclusion}

In conclusion, we reviewed the current progress of research on full-color III-nitride nanowire LEDs. In this reviewpaper, various nanowire growth techniques such as top-down and bottom-up approaches, and the device fabrication methods were discussed. It is reported that the III-nitride nanowires are grown on $\mathrm{Si}$ substrate which is CMOS compatible. Further, carrier dynamics and loss mechanisms of nanowire LEDs were studied as well as different nanowire LED structures with improved device performance were reported. It is presented that nanowire LEDs exhibit a better performance in terms of efficiency, output power, low dislocation density, and reduced manufacturing cost than conventional planar devices. Optical tunability in the entire visible spectrum is possible with nanowire LEDs and the blue, green, yellow, orange, and red color LEDs were demonstrated. Moreover, phosphor-free $\mathrm{cool} /$ warm white color nanowire LEDs were reported. The potential applications of these devices include smart lighting, micro-display, aviation lighting, visible light communication [140, 141], water splitting [142, 143], food processing/horticulture $[144,145]$, photo-therapy/medical diagnostics [146], and many more. Especially, device miniaturization and efficiency of III nitride nanowire LEDs are suitable for micro-display applications and competitive to current LCD and OLED technologies.

\section{Acknowledgement}

This work is being supported by the National Science Foundation under grant number ECCS194431, New Jersey Institute of Technology (NJIT) and the Instrument Usage Seed Grant from Otto H. York Center at NJIT.

\section{References}

[1] Ramesh, V., Kikuchi, A., Kishino, K., Funato, M., \& Kawakami, Y. (2010). Strain relaxation effect by nanotexturing InGaN/GaN multiple quantum well. J. Appl. Phys., 107(11), 114303.

[2] Chiu, C. H., et al. (2007). Fabrication of $\mathrm{InGaN} / \mathrm{GaN}$ nanorod light-emitting diodes with self-assembled $\mathrm{Ni}$ metal islands. Nanotechnology, 18(44), 445201.

[3] Bai, J., Wang, Q., \& Wang, T. (2012). Characterization of InGaN-based nanorod light emitting diodes with different indium compositions. J. Appl. Phys., 111(11), 113103.

[4] Li, Q., et al. (2011). Optical performance of top-down fabricated $\mathrm{InGaN} /$ GaN nanorod light emitting diode arrays. Opt. Express, 19(25), 25528.

[5] Bae, S.-Y., Kong, D.-J., Lee, J.-Y., Seo, D.-J., \& Lee, D.-S. (2013). Size-controlled InGaN/GaN nanorod array fabrication and optical characterization. Opt. Express, 21(14), 16854.

[6] Harmand, J. C., Patriarche, G., PereLaperne, N., Merat-Combes, M. N., Travers, L., \& Glas, F. (2005). Analysis of vapor-liquid-solid mechanism in 
Au-assisted GaAs nanowire growth. Appl. Phys. Lett., 87(20), 203101.

[7] Cai, Z., Garzon, S., Chandrashekhar, M. V. S., Webb, R. A., \& Koley, G. (2008). Synthesis and properties of high-quality $\mathrm{InN}$ nanowires and nanonetworks. J. Electron. Mater., 37(5), 585.

[8] Gottschalch, V., Wagner, G., Bauer, J., Paetzelt, H., \& Shirnow, M. (2008). VLS growth of GaN nanowires on various substrates. J Cryst. Growth, 310(23), 5123.

[9] Stern, E., et al. (2005). Electrical characterization of single GaN nanowires. Nanotechnology, 16(12), 2941.

[10] Kuykendall, T., Ulrich, P., Aloni, S., \& Yang, P. (2007). Complete composition tunability of InGaN nanowires using a combinatorial approach. Nat. Mater., 6(12), 951.

[11] Lauhon, L. J., Gudiksen, M. S., \& Lieber, C. M. (2004). Semiconductor nanowire heterostructures. Philos. Trans. R. Soc. London, Ser. A, 362(1819), 1247.

[12] Lauhon, L. J., Gudiksen, M. S., Wang, D., \& Lieber, C. M. (2002). Epitaxial coreshell and core-multishell nanowire heterostructures. Nature, 420(6911), 57.

[13] Ristic, J., et al. (2008). On the mechanisms of spontaneous growth of III-nitride nanocolumns by plasma-assisted molecular beam epitaxy. J. Cryst. Growth, $310(18), 4035$.

[14] Vajpeyi, A. P., et al. (2009). Spontaneous growth of III-nitride nanowires on Si by molecular beam epitaxy. Microelectron. Eng., 86(4-6), 812.

[15] Zhang, J., Peng, X. S., Wang, X. F., Wang, Y. W., \& Zhang, L. D. (2001). Micro-Raman investigation of GaN nanowires prepared by direct reaction Ga with NH3. Chem. Phys. Lett., 345, 372 .

[16] Kishino, K., Hoshino, T., Ishizawa, S., \& Kikuchi, A. (2008). Selective-area growth of GaN nanocolumns on titanium-maskpatterned silicon (111) substrates by RFplasma-assisted molecular-beam epitaxy. Electronics Letters, 44(13), 819.

[17] Hersee, S. D., et al. (2009). GaN nanowire light emitting diodes based on templated and scalable nanowire growth process. Electronics Letters, 45(1), 75 .

[18] Schumann, T., Gotschke, T., Limbach, F., Stoica, T., \& Calarco, R. (2011). Selectivearea catalyst-free MBE growth of $\mathrm{GaN}$ nanowires using a patterned oxide layer. (in eng), Nanotechnology, 22(9), 095603.

[19] Bengoechea-Encabo, A., et al. (2011). Understanding the selective area growth of GaN nanocolumns by MBE using $\mathrm{Ti}$ nanomasks. J. Cryst. Growth, 325(1), 89.

[20] Kamimura, J., Kishino, K., \& Kikuchi, A. (2012). Photoluminescence properties of selectively grown InN microcrystals. Phys. status solidi RRL, 6(4), 157.

[21] Hersee, S. D., Sun, X., \& Wang, X. (2006). The controlled growth of GaN nanowires. Nano Lett., 6, 1808.

[22] Chen, Y.-S., et al. (2009). Threading dislocation evolution in patterned $\mathrm{GaN}$ nanocolumn growth and coalescence overgrowth. J. Appl. Phys., 106(2), 023521.

[23] Bergbauer, W., et al. (2010). Continuousflux MOVPE growth of position-controlled $\mathrm{N}$-face GaN nanorods and embedded InGaN quantum wells. Nanotechnology, 21(30), 305201.

[24] Liu, C., et al. (2010). Variations in mechanisms of selective area growth of $\mathrm{GaN}$ on nano-patterned substrates by MOVPE. Phys. status solidi c, 7(1), 32 .

[25] Bertness, K. A., et al. (2010). Controlled nucleation of $\mathrm{GaN}$ nanowires grown with molecular beam epitaxy. Adv. Funct. Mater., 20(17), 2911.

[26] Zhang, L., Teng, C.-H., Hill, T. A., Lee, L.-K., Ku, P.-C., \& Deng, H. (2013). Single photon emission from site-controlled 
InGaN/GaN quantum dots. Appl. Phys. Lett., 103(19), 192114.

[27] Wang, X., et al. (2014). Ultrafast. superhigh gain visible-blind UV detector and optical logic gates based on nonpolar aaxial GaN nanowire. Nanoscale, 6(20), 12009.

[28] Wang, X., et al. (2012). Polarity control in $3 \mathrm{D}$ GaN structures grown by selective area MOVPE. Cryst. Growth Des., 12(5), 2552 .

[29] Li, S. F., et al. (2011). Polarity and its influence on growth mechanism during MOVPE growth of GaN sub-micrometer rods. Cryst. Growth Des., 11(5), 1573.

[30] Wang, X., Sun, X., Fairchild, M., \& Hersee, S. D. (2006). Fabrication of GaN nanowire arrays by confined epitaxy. Appl. Phys. Lett., 89(23), 233115.

[31] Choi, K., Arita, M., \& Arakawa, Y. (2012). Selective-area growth of thin GaN nanowires by MOCVD. J. Cryst. Growth, 357,58 .

[32] Wang, X., et al. (2013). Continuous-Flow MOVPE of Ga-Polar GaN Column Arrays and Core-Shell LED Structures. Crystal Growth \& Design, 13(8), 3475.

[33] Gacevic, Z., Sanchez, Gomez, \& Calleja, E. (2015). Formation mechanisms of GaN nanowires grown by selective area growth homoepitaxy. Nano Lett, 15(2), 1117.

[34] Hsieh, M. Y., Wang, C. Y., Chen, L. Y., Ke, M. Y., \& Huang, J. J. (2008). InGaNGaN nanorod light emitting arrays fabricated by silica nanomasks. IEEE J. Quantum Electron., 44(5-6), 468.

[35] Wang, C. Y., et al. (2008). GaN nanorod light emitting diode arrays with a nearly constant electroluminescent peak wavelength. Opt. Express, 16(14), 10549.

[36] Nguyen, H. P. T., et al. (2011). p-Type Modulation Doped InGaN/GaN Dot-in-aWire White-Light-Emitting Diodes Monolithically Grown on $\mathrm{Si}(111)$. Nano Lett., 11(5), 1919.
[37] Waag, A., et al. (2011). The nanorod approach: GaN NanoLEDs for solid state lighting. Phys. Status Solidi C, 8(7-8), 2296 .

[38] Chen, L. Y., et al. (2010). High performance InGaN/GaN nanorod light emitting diode arrays fabricated by nanosphere lithography and chemical mechanical polishing processes. Opt. Express 18(8), 7664.

[39] Kikuchi, A., Kawai, M., Tada, M., \& Kishino, K. (2004). InGaN/GaN multiple quantum disk nanocolumn light-emitting diodes grown on (111)Si substrate. Jpn. J. Appl. Phys., Part 2, 43(12A), L1524.

[40] Philip, M. R., Choudhary, D. D., Djavid, M., Le, K. Q., Piao, J., \& Nguyen, H. P. T. (2017). High efficiency green/yellow and red InGaN/AlGaN nanowire lightemitting diodes grown by molecular beam epitaxy. Journal of Science: Advanced Materials and Devices, 2(2), 150.

[41] Chang, C., Chen, L., Huang, L., Wang, Y., Lu, T., \& Huang, J. J. (2012). Effects of Strains and Defects on the Internal Quantum Efficiency of InGaN/GaN Nanorod Light Emitting Diodes. IEEE J. Quantum Electron., 48(4), 551.

[42] Liang-Yi, C., Chi-Kang, L., Jin-Yi, T., Li-Chuan, H., Yuh-Renn, W., \& Jang, Jian (2013). On the Efficiency Decrease of the GaN Light-Emitting Nanorod Arrays. IEEE J. Quantum Electron., 49(2), 224.

[43] Min-Yung, K., et al. (2009). Application of nanosphere lithography to LED surface texturing and to the fabrication of nanorod LED arrays. IEEE J. Quantum Electron., 15(4), 1242.

[44] Kim, H.-M., et al. (2004). High-brightness light emitting diodes using dislocationfree indium gallium nitride/gallium nitride multiquantum-well nanorod arrays. Nano Lett., 4(6), 1059.

[45] Chang, C. Y., et al. (2006). Electrical transport properties of single $\mathrm{GaN}$ and InN nanowires. J. Electron. Mater., 35(4), 738. 
[46] Songmuang, R., et al. (2011). Strong suppression of internal electric field in $\mathrm{GaN} / \mathrm{AlGaN}$ multi-layer quantum dots in nanowires. Appl. Phys. Lett., 99(14), 141914.

[47] Pendyala, C., et al. (2012). Nanowires as semi-rigid substrates for growth of thick, InxGa1-xN $(x>0.4)$ epi-layers without phase segregation for photoelectrochemical water splitting. Nanoscale, 4(20), 6269.

[48] Motayed, A., Davydov, A. V., He, M., Mohammad, S. N., \& Melngailis, J. (2007). $365 \mathrm{~nm}$ operation of n-nanowire/pgallium nitride homojunction light emitting diodes. Appl. Phys. Lett., 90(18), 183120 .

[49] Van, C. G., de Walle, \& Segev, D. (2007). Microscopic origins of surface states on nitride surfaces. J. Appl. Phys., 101(8), 081704 .

[50] Nguyen, H. P. T., Cui, K., Zhang, S., Fathololoumi, S., \& Mi, Z. (2011). Full-color InGaN/GaN dot-in-a-wire light emitting diodes on silicon. Nanotechnology, 22(44), 445202.

[51] Nguyen, H. P. T., et al. (2012). Controlling electron overflow in phosphor-free In$\mathrm{GaN} / \mathrm{GaN}$ nanowire white light-emitting diodes. Nano Lett., 12(3), 1317.

[52] Lin, B.-C., et al. (2014). Hole injection and electron overflow improvement in $\mathrm{InGaN} /-$ GaN light-emitting diodes by a tapered AlGaN electron blocking layer. Opt. Express, 22(1), 463.

[53] Wei, T., et al. (2014). Investigation of efficiency and droop behavior comparison for InGaN/GaN super wide-well light emitting diodes grown on different substrates. IEEE Photonics J, 6(6), 1.

[54] Bertazzi, F., Zhou, X., Goano, M., Ghione, G., \& Bellotti, E. (2013). Auger recombination in $\mathrm{InGaN} / \mathrm{GaN}$ quantum wells: A full-Brillouin-zone study. Appl. Phys. Lett., 103(8), 081106.
[55] Shen, Y. C., Mueller, G. O., Watanabe, S., Gardner, N. F., Munkholm, A., \& Krames, M. R. (2007). Auger recombination in InGaN measured by photoluminescence. Appl. Phys. Lett., 91, 141101.

[56] Zhang, M., Bhattacharya, P., Singh, J., \& Hinckley, J. (2009). Direct measurement of auger recombination in $\mathrm{In} 0.1 \mathrm{Ga} 0.9 \mathrm{~N} / \mathrm{GaN}$ quantum wells and its impact on the efficiency of In0.1Ga0.9N/GaN multiple quantum well light emitting diodes. Appl. Phys. Lett., 95(20), 201108.

[57] Kioupakis, E., Rinke, P., Delaney, K. T., \& Walle, Van de (2011). Indirect auger recombination as a cause of efficiency droop in nitride light-emitting diodes. Appl. Phys. Lett., 98(16), 161107.

[58] Iveland, J., Martinelli, L., Peretti, J., Speck, J. S., \& Weisbuch, C. (2013). Direct measurement of Auger electrons emitted from a semiconductor light-emitting diode under electrical injection: identification of the dominant mechanism for efficiency droop. Phys. Rev. Lett, 110(17), 177406 .

[59] Renwick, P., Tang, H., Bai, J., \& Wang, T. (2012). Reduced longitudinal optical phonon-exciton interaction in InGaN/GaN nanorod structures. Appl. Phys. Lett., 100(18), 182105.

[60] Guo, W., Zhang, M., Bhattacharya, P., \& Heo, J. (2011). Auger recombination in III-nitride nanowires and its effect on nanowire light-emitting diode characteristics. Nano Lett., 11(4), 1434.

[61] Nguyen, H. P. T., Djavid, M., Cui, K., \& Mi, Z. (2012). Temperature-dependent nonradiative recombination processes in GaN-based nanowire white-light-emitting diodes on silicon. Nanotechnology, 23(19), 194012.

[62] Ryu, M. Y., et al. (2000). Photoluminescence study of InGaN/GaN double quantum wells with varying barrier widths. Journal of Korean Physical Society, 37(4), 387. 
[63] De, S., et al. (2012). Quantum-confined stark effect in localized luminescent centers within InGaN/GaN quantum-well based light emitting diodes. Appl. Phys. Lett., 101(12), 121919.

[64] Zhu, J., et al. (2011). The investigation on carrier distribution in InGaN/GaN multiple quantum well layers. J. Appl. Phys., 109(9), 093117.

[65] Liu, J., Ryou, J.-H., Dupuis, R., Han, J., Shen, G., \& Wang, H. (2008). Barrier effect on hole transport and carrier distribution in InGaN/GaN multiple quantum well visible light-emitting diodes. Appl. Phys. Lett., 93(2), 1102.

[66] Xie, J. Q., Ni, X. F., Fan, Q., Shimada, R., Ozgur, U., \& Morkoc, H. (2008). On the efficiency droop in InGaN multiple quantum well blue light emitting diodes and its reduction with p-doped quantum well barriers. Appl. Phys. Lett., 93(12), 121107.

[67] Rozhansky, I., \& Zakheim, D. (2006). Analysis of the causes of the decrease in the electroluminescence efficiency of AlGaInN light-emitting-diode heterostructures at high pumping density. Semiconductors, 40(7), 839.

[68] Ni, X., Fan, Q., Shimada, R., Özgür, Ü., \& Morkoç, H. (2008). Reduction of efficiency droop in InGaN light emitting diodes by coupled quantum wells. Appl. Phys. Lett., 93(17), 171113.

[69] Tsai, M.-C., Yen, S.-H., Lu, Y.-C., \& Kuo, Y.-K. (2011). Numerical study of blue InGaN light-emitting diodes with varied barrier thicknesses. IEEE Photonics Technol. Lett., IEEE, 23(2), 76.

[70] Piprek, J., \& Li, Z. M. (2010). Electron leakage effects on the efficiency droop in GaN-based light-emitting diodes. IEEE Numerical Simulation of Optoelectronics Devices, 89 .

[71] Ni, X., et al. (2010). Hot electron effects on efficiency degradation in InGaN light emitting diodes and designs to mitigate them. J. Appl. Phys., 108(3), 033112.
[72] Pope, I., Smowton, P. M., Blood, P., Thomson, J. D., Kappers, M., \& Humphreys, C. (2003). Carrier leakage in InGaN quantum well light-emitting diodes emitting at $480 \mathrm{~nm}$. Appl. Phys. Lett, $82(17), 2755$.

[73] Singh, S., Robidas, D., Rohila, N., Pal, S., \& Dhanavantri, C. (2010). Effect of electron blocking layer on efficiency droop in blue InGaN/GaN based lightemitting diodes. Optoelectronics and Advanced Materials-Rapid Communications, 4(23), 1106.

[74] Özgür, Ü., et al. (2010). Ballistic transport in InGaN-based LEDs: impact on efficiency. Semicond. Sci. Technol., 26(1), 014022 .

[75] Vampola, K. J., Iza, M., Keller, S., DenBaars, S. P., \& Nakamura, S. ( Measurement of electron overflow in $450 \mathrm{~nm}$ InGaN light-emitting diode structures. Appl. Phys. Lett., 94(6), 061116.

[76] Djavid, M., Nguyen, H., Zhang, S., Cui, K., Fan, S., \& Mi, Z. (2014). Tunnel injection $\mathrm{InGaN} / \mathrm{GaN}$ dot-in-a-wire whitelight-emitting diodes. Semicond. Sci. Technol., 29(8), 085009.

[77] Moram, M., et al. (2009). On the origin of threading dislocations in GaN films. J. Appl. Phys., 106(7), 073513.

[78] Narayanan, V., Lorenz, K., Kim, W., \& Mahajan, S. (2001). Origins of threading dislocations in $\mathrm{GaN}$ epitaxial layers grown on sapphire by metalorganic chemical vapor deposition. Appl. Phys. Lett., 78(11), 1544 .

[79] Rao, D. S., Beanland, R., Kappers, M., Zhu, D., \& Humphreys, C. (2010). Lattice distortions in GaN thin films on (0001) sapphire. Journal of Physics: Conference Series, 209(1), 012022.

[80] Guo, W., Banerjee, A., Bhattacharya, P., \& Ooi, B. S. (2011). InGaN/GaN disk-innanowire white light emitting diodes on (001) silicon. Appl. Phys. Lett., 98(19), 193102. 
[81] Wang, C., et al. (2010). Temperaturedependent electroluminescence efficiency in blue InGaN-GaN light-emitting diodes with different well widths. IEEE Photonics Technol. Lett., 22(4), 236.

[82] Kim, M. H., et al. (2007). Origin of efficiency droop in GaN-based light-emitting diodes. Appl. Phys. Lett., 91, 183507.

[83] Zhang, S., et al. (2013). On the efficiency droop of top-down etched InGaN/GaN nanorod light emitting diodes under optical pumping. AIP Adv., 3(8), 082103.

[84] Jahangir, S., Mandl, M., Strassburg, M., \& Bhattacharya, P. (2013). Molecular beam epitaxial growth and optical properties of red-emitting $(\lambda=650 \mathrm{~nm}) \mathrm{InGaN} /-$ GaN disks-in-nanowires on silicon. Appl. Phys. Lett., 102(7), 071101.

[85] Nguyen, H. P. T., et al. (2013). Breaking the carrier injection bottleneck of phosphor-free nanowire white lightemitting diodes. Nano Lett., 13(11), 5437.

[86] Martinez, G., Curiel, M., Skromme, B., \& Molnar, R. (2000). Surface recombination and sulfide passivation of GaN. J. Electron. Mater., 29(3), 325.

[87] Hartensveld, M., Ouin, G., Liu, C., \& Zhang, J. (2019). Effect of KOH passivation for top-down fabricated InGaN nanowire light emitting diodes. J. Appl. Phys., 126(18), 183102.

[88] Chen, W. D., Li, X. Q., Duan, L. H., Xie, X. L., \& Cui, Y. D. (1996). Photoluminescence enhancement of (NH4)2Sx passivated InP surface by rapid thermal annealing. Appl. Surf. Sci., 100, 592.

[89] Chevtchenko, S. A., et al. (2007). Study of $\mathrm{SiNx}$ and $\mathrm{SiO} 2$ passivation of GaN surfaces. J. Appl. Phys., 101(11), 113709.

[90] Tajik, N., Haapamaki, C. M., \& LaPierre, R. R. (2012). Photoluminescence model of sulfur passivated p-InP nanowires. Nanotechnology, 23(31), 315703.
[91] Chang, C.-C., et al. (2012). Electrical and optical characterization of surface passivation in GaAs nanowires. Nano Lett., 12(9), 4484.

[92] Joyce, H. J., et al. (2013). Electronic properties of GaAs, InAs and InP nanowires studied by terahertz spectroscopy. Nanotechnology, 24(21), 214006.

[93] Parkinson, P., et al. (2009). Carrier lifetime and mobility enhancement in nearly defect-free core- shell nanowires measured using time-resolved terahertz spectroscopy. Nano Lett., 9(9), 3349.

[94] Zhang, S., et al. (2014). On the carrier injection efficiency and thermal property of In GaN/GaN axial nanowire light emitting diodes. Quantum Electronics, IEEE J. Quantum Electron., 50(6), 483.

[95] Schlager, J. B., Bertness, K. A., Blanchard, P. T., Robins, L. H., Roshko, A., \& Sanford, N. A. (2008). Steady-state and time-resolved photoluminescence from relaxed and strained GaN nanowires grown by catalyst-free molecular-beam epitaxy. J. Appl. Phys., 103(12), 124309.

[96] Armstrong, A., Li, Q., Lin, Y., Talin, A., \& Wang, G. (2010). GaN nanowire surface state observed using deep level optical spectroscopy. Appl. Phys. Lett., 96(16), 163106.

[97] Nguyen, H. P. T., et al. (2015). Engineering the Carrier Dynamics of InGaN Nanowire White Light-Emitting Diodes by Distributed p-AlGaN Electron Blocking Layers. Sci. Rep., Article 5, 7744.

[98] Hahn, C., et al. (2011). Epitaxial Growth of InGaN Nanowire Arrays for Light Emitting Diodes. Acs Nano, 5(5), 3970.

[99] Guo, W., Zhang, M., Banerjee, A., \& Bhattacharya, P. (2010). Catalyst-Free InGaN/GaN Nanowire Light Emitting Diodes Grown on (001) Silicon by Molecular Beam Epitaxy. Nano Lett., 10(9), 3355.

[100] Armitage, R., \& Tsubaki, K. (2010). Multicolour luminescence from InGaN quantum wells grown over GaN nanowire arrays 
by molecular-beam epitaxy. Nanotechnology, 21(19), 195202.

[101] Boney, C., et al. (2010). Growth and characterization of InGaN for photovoltoic devices. 35th IEEE Photovoltaic Specialists Conference.

[102] Tchernycheva, M., et al. (2014). In$\mathrm{GaN} / \mathrm{GaN}$ core-shell single nanowire light emitting diodes with graphene-based pcontact. Nano Lett., 14(5), 2456.

[103] Ra, Y.-H., Navamathavan, R., Yoo, H.-I., \& Lee, C.-R. (2014). Single nanowire lightemitting diodes using uniaxial and coaxial In GaN/GaN multiple quantum wells synthesized by metalorganic chemical vapor deposition. Nano Lett., 14(3), 1537.

[104] Wang, R., Liu, X., Shih, I., \& Mi, Z. (2015). High efficiency, full-color AlInGaN quaternary nanowire light emitting diodes with spontaneous core-shell structures on Si. Appl. Phys. Lett., 106(26), 261104.

[105] Simon, J., Protasenko, V., Lian, C., Xing, H., \& Jena, D. (2010). PolarizationInduced Hole Doping in Wide-Band-Gap Uniaxial Semiconductor Heterostructures. Science, 327(5961), 60.

[106] Carnevale, S. D., Kent, T. F., Phillips, P. J., Mills, M. J., Rajan, S., \& Myers, R. C. (2012). Polarization-Induced pn Diodes in Wide-Band-Gap Nanowires with Ultraviolet Electroluminescence. Nano Lett., 12(2), 915 .

[107] Carnevale, S. D., et al. (2013). Mixed Polarity in Polarization-Induced $\mathrm{p}-\mathrm{n}$ Junction Nanowire Light-Emitting Diodes. Nano Lett., 13(7), 3029.

[108] Sadaf, S., Ra, Y., Szkopek, T., \& Mi, Z. (2016). Monolithically integrated metal/semiconductor tunnel junction nanowire light-emitting diodes. Nano Lett., 16(2), 1076.

[109] Sadaf, S., Ra, Y.-H., Nguyen, H., Djavid, M., \& Mi, Z. (2015). Alternating-current In GaN/GaN tunnel junction nanowire white-light emitting diodes. Nano Lett., 15(10), 6696 .
[110] Tan, B. S., Yuan, S., \& Kang, X. J. (2004). Performance enhancement of InGaN light-emitting diodes by laser lift-off and transfer from sapphire to copper substrate. Appl. Phys. Lett., 84(15), 2757.

[111] Ng, Tien Khee, et al. (2014). Red to Near-Infrared Emission from $\mathrm{InGaN} /-$ GaN Quantum-Disks-in-Nanowires LED, in CLEO: 2014, OSA Technical Digest (online), Optical Society of America.

[112] Nguyen, H. P. T., Wang, Q., \& Mi, Z. (2014). Phosphor-Free InGaN/GaN Dotin-a-Wire White Light-Emitting Diodes on Copper Substrates. J. Electron. Mater., $43(4), 868$.

[113] Philip, Rajan, et al. (2017). Fabrication of Phosphor-Free III-Nitride Nanowire Light-Emitting Diodes on Metal Substrates for Flexible Photonics. ACS Omega, 2(9), 5708.

[114] Zhao, C., et al. (2016). Facile Formation of High-Quality InGaN/GaN QuantumDisks-in-Nanowires on Bulk-Metal Substrates for High-Power Light-Emitters. Nano Lett., 16(2), 1056.

[115] Zhao, C., et al. (2016). Droop-Free. Reliable, and High-Power InGaN/GaN Nanowire Light-Emitting Diodes for Monolithic Metal-Optoelectronics. Nano Lett., 16(7), 4616.

[116] Oh, J. H., Yang, S. J., \& Do, Y. R. (2014). Healthy, natural, efficient and tunable lighting: four-package white LEDs for optimizing the circadian effect, color quality and vision performance. Ligh Sci. Appl., 3(2), e141.

[117] Krames, M. R., et al. (2007). Status and future of high-power light-emitting diodes for solid-state lighting. J. Disp. Technol., $3(2), 160$.

[118] Kölper, C., Sabathil, M., Römer, F., Mandl, M., Strassburg, M., \& Witzigmann, B. (2012). Core-shell InGaN nanorod light emitting diodes: Electronic and optical device properties. Phys. status solidi A, 209(11), 2304. 
[119] Wang, R., Nguyen, H., Connie, A. T., Lee, J., Shih, I., \& Mi, Z. (2014). Colortunable, phosphor-free InGaN nanowire light-emitting diode arrays monolithically integrated on silicon. Opt. Express, 22(107), A1768.

[120] Sekiguchi, H., Kishino, K., \& Kikuchi, A. (2010). Emission color control from blue to red with nanocolumn diameter of In$\mathrm{GaN} / \mathrm{GaN}$ nanocolumn arrays grown on same substrate. Appl. Phys. Lett., 96(23), 231104.

[121] Sekiguchi, H., Kishino, K., \& Kikuchi, A. (2008). Ti-mask selective-area growth of GaN by RF-plasma-assisted molecularbeam epitaxy for fabricating regularly arranged InGaN/GaN nanocolumns. Appl. Phys. Express, 1(12), 124002.

[122] Albert, S., Bengoechea-Encabo, A., Kong, X., Sanchez-Garcia, M., Calleja, E., \& Trampert, A. (2013). Monolithic integration of InGaN segments emitting in the blue, green, and red spectral range in single ordered nanocolumns. Appl. Phys. Lett., 102(18), 181103.

[123] Zhao, S., Kibria, M., Wang, Q., Nguyen, H., \& Mi, Z. (2013). Growth of large-scale vertically aligned $\mathrm{GaN}$ nanowires and their heterostructures with high uniformity on $\mathrm{SiO} \times$ by catalyst-free molecular beam epitaxy. Nanoscale, 5(12), 5283 .

[124] Park, Y., Jahangir, S., Park, Y., Bhattacharya, P., \& Heo, J. (2015). InGaN/GaN nanowires grown on $\mathrm{SiO} 2$ and light emitting diodes with low turn on voltages. Opt. Express, 23(11), A650.

[125] Schubert, M. F., et al. (2008). Polarization-matched GaInN/AlGaInN multi-quantum-well light-emitting diodes with reduced efficiency droop. Appl. Phys. Lett., 93(4), 041102.

[126] Monemar, B., \& Sernelius, B. E. (2007). Defect related issues in the current rolloff in InGaN based light emitting diodes. Appl. Phys. Lett., 91(18), 181103.
[127] Setlur, A. A., et al. (2010). Energyefficient. high-color-rendering LED lamps using oxyfluoride and fluoride phosphors. Chem. Mater., 22(13), 4076.

[128] Sommer, C., Hartmann, P., Pachler, P., Hoschopf, H., \& Wenzl, F. P. (2012). White light quality of phosphor converted light-emitting diodes: A phosphor materials perspective of view. J. Alloys Compd., 520, 146-152.

[129] Haitz, R., \& Tsao, J. Y. (2011). Solid-state lighting:'The case'10 years after and future prospects. Phys. Status Solidi A, 208(1), 17.

[130] Kölper, C., Sabathil, M., Mandl, M., Strassburg, M., \& Witzigmann, B. (2012). All-InGaN phosphorless white light emitting diodes: an efficiency estimation. J. Lightwave Technol, 30(17), 2853.

[131] Qian, F., Gradecak, S., Li, Y., Wen, C., \& Lieber, C. (2005). Core/multishell nanowire heterostructures as multicolor, high-efficiency light-emitting diodes. Nano Lett., 5(11), 2287.

[132] Bui, H. Q. T., et al. (2019). Full-Color InGaN/AlGaN Nanowire Micro LightEmitting Diodes Grown by Molecular Beam Epitaxy: A Promising Candidate for Next Generation Micro Displays. Micromachines, 10(8), 492.

[133] Nami, M., et al. (2018). Carrier dynamics and electro-optical characterization of high-performance GaN/InGaN core-shell nanowire light-emitting diodes. Sci. Rep., $8(1), 501$.

[134] Nami, M., et al. (2019). Electrically Injected $\mathrm{GHz}$-Class GaN/InGaN Core-Shell Nanowire-Based $\mu$ LEDs: Carrier Dynamics and Nanoscale Homogeneity. ACS Photonics, 6(7), 1618.

[135] Hong, C. C., Ann, H., Wu, C. Y., \& Gwo, S. (2009). Strong green photoluminescence from InxGa1-xN/GaN nanorod arrays. Opt. Express, 17(20), 17227. 
[136] Chang, Y. L., Wang, J. L., Li, F., \& Mi, Z. (2010). High efficiency green, yellow, and amber emission from InGaN/GaN dot-ina-wire heterostructures on $\mathrm{Si}(111)$. Appl. Phys. Lett., 96(1), 013106.

[137] Trung, Pham, Shaofei, Z., Kai, C., Korinek, A., Botton, G. A., \& Mi, Z. (2012). High-Efficiency InGaN/GaN Dotin-a-Wire Red Light-Emitting Diodes. IEEE Photonics Technol. Lett., 24(4), 321.

[138] Hong, Y. J., et al. (2011). Visible-ColorTunable Light-Emitting Diodes. Adv. Mater., 23(29), 3284.

[139] Lin, H.-W., Lu, Y.-J., Chen, H.-Y., Lee, H.-M., \& Gwo, S. (2010). InGaN/GaN nanorod array white light-emitting diode. Appl. Phys. Lett., 97(7), 073101.

[140] Du, C., et al. (2016). Tuning carrier lifetime in InGaN/GaN LEDs via strain compensation for high-speed visible light communication. Sci. Rep., 6, 37132.

[141] Philip, M. R., Bui, T. H. Q., Djavid, M., Bhuyian, M. N., Vu, P., \& Nguyen, H. P. T. (2018). Phosphor-free III-nitride nanowire white-light-emitting diodes for visible light communication. in Active and Passive Smart Structures and Integrated Systems XII, 10595, 105953 I.

[142] Ebaid, M., Min, J.-W., Zhao, C., Ng, T. K., Idriss, H., \& Ooi, B. S. (2018). Water splitting to hydrogen over epitaxially grown InGaN nanowires on a metallic titanium/silicon template: reduced interfacial transfer resistance and improved stability to hydrogen. J. Mater. Chem. A, 6(16), 6922 .

[143] Kibria, M., et al. (2015). Visible lightdriven efficient overall water splitting using p-type metal-nitride nanowire arrays. Nat. commun., 6, 6797.

[144] Ishikawa, T., \& Isowa, K. (2012). Cultivation of microalgae for live food under white light-emitting diodes (LEDs). Journal of Fisheries Technology, 4(2), 51.
[145] Lefcourt, A. M., Wiederoder, M. S., Liu, N. T., Kim, M. S., \& Lo, Y. M. (2013). Development of a portable hyperspectral imaging system for monitoring the efficacy of sanitation procedures in food processing facilities. J. Food Eng., 117(1), 59.

[146] Chaves, M. E. D. A., Araújo, A. R. d., Piancastelli, A. C. C., \& Pinotti, M. (2014). Effects of low-power light therapy on wound healing: LASER x LED. Anais brasileiros de dermatologia, 89(4), 616.

\section{About Authors}

Ravi Teja VELPULA received Master of Technology in Photonics Science and Engineering from Indian Institute of Technology (IIT) Kanpur, India in 2017 and was awarded GATE scholarship during the two years of his Master's program. He is currently working towards earning his Ph.D. degree in Electrical Engineering at New Jersey Institute of Technology (NJIT), USA with emphasis on "III-nitride nanowire structures for photonic applications" under Prof. Hieu Nguyen's supervision. His research interest lies on modeling, fabrication and characterization III-nitride based LEDs/ LASERs. He is the author/co-author of six peer-reviewed articles in high impact journals and one conference paper. In addition, he serves as reviewer for OSA Applied Optics journal.

Barsha JAIN is currently working towards earning her Doctorate in Electrical Engineering with emphasis on "III-nitride nanowire structures for photonic and electronic applications" under Prof. Hieu Nguyen's supervision in the Electrical and Computer Engineering Department at NJIT. Her research interest lies on simulation, fabrication and characterization of III-nitride based light emitters and non-volatile memory devices such as RRAM and PCM devices and authored/co-authored six peer-reviewed articles in high impact journals and three conference papers. In 2017, she received Master of Technology in Electrical Engineering with emphasis on VLSI Design from Atal Bihari Vajpayee Indian Institute of Information Technology and Management 
(ABV IIITM) Gwalior and was awarded GATE scholarship during the two years of her Master's program.

Ha Quoc Thang BUI is currently third-year Ph.D. candidate in the Department of Electrical and Computer Engineering at NJIT. He graduated with a Bachelor's degree in Physics from the Vietnam National University - University of Science in 2005, and a Master's degree in Nanomaterials and Nanodevices from Vietnam National University - University of Engineering and Technology. His research focuses on IIInitride materials for optoelectronic devices such as light-emitting diodes and their applications. $\mathrm{He}$ is an author/co-author of 8 peer-reviewed papers.

Hieu Pham Trung NGUYEN received his Ph.D. degree in Electrical Engineering from McGill University, Canada in 2012. He is an assistant professor of Electrical and Computer Engineering at NJIT. He is the Director of the Nano-Optoelectronics Materials and Devices Laboratory at NJIT. His current research interests include design, molecular beam epitaxial growth, fabrication, and characterization of nanowire heterostructures for high performance nano-optoelectronic devices including light-emitting diodes, lasers, photodetectors, solar fuels, and solar cells. Prof. Nguyen is a member of the IEEE, IEEE Photonics Society, the international society for optics and photonics (SPIE), and the American Chemical Society. He holds two U.S. patents, and is the author/coauthor of more than 65 journal articles and 100 conference presentations including several plenary and invited talks. $\mathrm{He}$ is a recipient of the SPIE scholarship in optics (2012), the outstanding paper award at the 28th North American Molecular Beam Epitaxy conference (2011), and the best student paper award (2nd place) at the IEEE photonics conference (2011). He received the 2019 Saul K. Fenster Innovation in Engineering Education Award for his innovative teaching effectiveness in engineering education. (This award recognizes a Newark College of Engineering full-time tenured/tenure-track faculty member who has demonstrated creativity and innovation in his or her teaching methods) and the prestigious 2020 U.S. National Science Foundation Career Award. 